\title{
Parallel-Correctness and Transferability for Conjunctive Queries
}

\author{
Tom J. Ameloot \\ Hasselt University
}

\author{
Gaetano Geck \\ TU Dortmund University
}

\author{
Bas Ketsman \\ Hasselt University
}

\author{
Frank Neven \\ Hasselt University
}

\author{
Thomas Schwentick \\ TU Dortmund University
}

\begin{abstract}
A dominant cost for query evaluation in modern massively distributed systems is the number of communication rounds. For this reason, there is a growing interest in single-round multiway join algorithms where data is first reshuffled over many servers and then evaluated in a parallel but communication-free way. The reshuffling itself is specified as a distribution policy. We introduce a correctness condition, called parallel-correctness, for the evaluation of queries w.r.t. a distribution policy. We study the complexity of parallel-correctness for conjunctive queries as well as transferability of parallelcorrectness between queries. We also investigate the complexity of transferability for certain families of distribution policies, including, for instance, the Hypercube distribution.
\end{abstract}

\section{Introduction}

In traditional database systems, the complexity of query processing for large datasets is mainly determined by the number of IO requests to external memory. A factor dominating complexity in modern massively distributed database systems, however, is the number of communication steps [7. Motivated by recent in-memory systems like Spark [1] and Shark [16], Koutris and Suciu introduced the massively parallel communication model (MPC) [14] where computation proceeds in a sequence of parallel steps each followed by global synchronization of all servers. In this model, evaluation of conjunctive queries [6, 14] and skyline queries [3] has been considered.

Of particular interest in the MPC model are the queries that can be evaluated in one round of communication. Recently, Beame, Koutris and Suciu [7] proved a matching upper and lower bound for the amount of communication needed to compute a full conjunctive query without self-joins in one communication round. The upper bound is provided by a randomized algorithm called Hypercube which uses a technique that can be traced back to Ganguli, Silberschatz, and Tsur [11] and is described in the context of map-reduce by Afrati and Ullman [4. The Hypercube algorithm evaluates a conjunctive query $\mathcal{Q}$ by first reshuffling the data over many servers and then evaluating $\mathcal{Q}$ at each server in a parallel but communication-free manner. The reshuffling is specified by a distribution policy (hereafter, called Hypercube distribution) and is based on the structure of $\mathcal{Q}$. In particular, the Hypercube distribution partitions the space of all complete valuations of $\mathcal{Q}$ over the computing servers in an instance independent way through hashing of domain values. A property of $\mathrm{Hy}-$ percube distributions is that for any instance $I$, the central execution of $\mathcal{Q}(I)$ always equals the union of the evaluations of $\mathcal{Q}$ at every computing node (or server) 1

In this paper, we introduce a general framework for reasoning about one-round evaluation algorithms under arbitrary distribution policies. Distribution poli-

\footnotetext{
${ }^{1}$ We note that, for a query $\mathcal{Q}$, there is no single Hypercube distribution but rather a family of distributions as the concrete instantiation depends on choices regarding the address space of servers.
} 
cies (formally defined in Section 2) are functions mapping input facts to sets of nodes (servers) in the network. We introduce the following correctness property for queries and distribution policies: a query $\mathcal{Q}$ is parallel-correct for a given distribution policy $\boldsymbol{P}$, when for any instance $I$, the evaluation of $\mathcal{Q}(I)$ equals the union of the evaluation of $\mathcal{Q}$ over the distribution of $I$ under policy $\boldsymbol{P}$. We focus on conjunctive queries and study the complexity of deciding parallelcorrectness. We show that the latter problem is equivalent to testing whether the facts in every minimal valuation of the conjunctive query are mapped to a same node in the network by the distribution policy. For various representations of distributions policies, we then show that testing parallel-correctness is in $\Pi_{2}^{P}$. We provide a matching lower bound via a reduction from the $\Pi_{2}^{P}$-complete $\Pi_{2}$-QBF-problem.

One-round evaluation algorithms, like Hypercube, redistribute data for the evaluation of every query. For scenarios where queries are executed in sequence, it makes sense to study cases where the same data distribution can be used to evaluate multiple queries. We formalize this as parallel-correctness transfer between queries. In particular, parallel-correctness transfers from $\mathcal{Q}$ to $\mathcal{Q}^{\prime}$ when $\mathcal{Q}^{\prime}$ is parallel-correct under every distribution policy for which $\mathcal{Q}$ is parallelcorrect. We characterize transferability for conjunctive queries by a (value-based) containment condition for minimal valuations of $\mathcal{Q}^{\prime}$ and $\mathcal{Q}$, and use this characterization to obtain a $\Pi_{3}^{P}$ upper bound for transferability. Again, we obtain a matching lower bound, this time via a reduction from the $\Pi_{3}^{P}$-complete $\Pi_{3}$-QBF-problem. We obtain a (presumably) better complexity, NP-completeness, in the case that $\mathcal{Q}$ is strongly minimal, i.e., when all its valuations are minimal. Examples of strongly minimal CQs include the full conjunctive queries and those without self-joins. At the heart of the upper bound proof lies the insight that the above mentioned valuebased inclusion w.r.t. minimal valuations reduces to a syntactic inclusion of $\mathcal{Q}^{\prime}$ in $\mathcal{Q}$ modulo a variable renaming when $\mathcal{Q}$ is strongly minimal. We obtain that deciding strong minimality is NP-complete as well.

Finally, we study parallel-correctness transfer from $\mathcal{Q}$ to $\mathcal{Q}^{\prime}$ w.r.t. a specific family of distribution policies $\mathcal{F}$ rather than the set of all distribution policies.
We show that it is NP-complete to decide whether $\mathcal{Q}^{\prime}$ is parallel-correct for a given family $\mathcal{F}$ if this family has the following two properties: it is $\mathcal{Q}$-generous (for each, not only for minimal, valuation of $\mathcal{Q}$, its facts occur at some node) and $\mathcal{Q}$-scattered (for every instance some distribution has, at every node, only facts from one valuation). It is easy to see that the family of Hypercube distributions for a given CQ $\mathcal{Q}$ satisfies these properties, which implies that deciding transferability for Hypercube distributions is NP-complete, as well.

We complete our framework by sketching a declarative specification formalism for distribution policies, illustrated with the specification of Hypercube distributions.

Due to space restrictions, many proofs are moved to an appendix.

Outline. We introduce the necessary definitions in Section 2, We study parallel-correctness in Section 3 and transferability in Section 4 . We examine families of distribution policies including the Hypercube distribution in Section 5. We conclude in Section 6 .

\section{Definitions}

Queries and instances. We assume an infinite set dom of data values that can be represented by strings over some fixed alphabet. A database schema $\mathcal{D}$ is a finite set of relation names $R$ where every $R$ has arity $\operatorname{ar}(R)$. We call $R(\mathbf{t})$ a fact when $R$ is a relation name and $\mathbf{t}$ a tuple in dom. We say that a fact $R\left(d_{1}, \ldots, d_{k}\right)$ is over a database schema $\mathcal{D}$ if $R \in \mathcal{D}$ and $\operatorname{ar}(R)=k$. By facts $(\mathcal{D})$, we denote the set of possible facts over schema $\mathcal{D}$. A (database) instance $I$ over $\mathcal{D}$ is a finite set of facts over $\mathcal{D}$. By adom $(I)$ we denote the set of data values occurring in $I$. A query $\mathcal{Q}$ over input schema $\mathcal{D}_{1}$ and output schema $\mathcal{D}_{2}$ is a generic mapping from instances over $\mathcal{D}_{1}$ to instances over $\mathcal{D}_{2}$. Genericity means that for every permutation $\pi$ of dom and every instance $I$, $\mathcal{Q}(\pi(I))=\pi(\mathcal{Q}(I))$

Conjunctive queries. Let var be the universe of variables, disjoint from dom. An atom is of the form 
$R(\mathbf{x})$, where $R$ is a relation name and $\mathbf{x}$ is a tuple of variables in var. We say that $R\left(x_{1}, \ldots, x_{k}\right)$ is an atom over schema $\mathcal{D}$ if $R \in \mathcal{D}$ and $k=\operatorname{ar}(R)$.

A conjunctive query $\mathcal{Q}(\mathrm{CQ})$ over input schema $\mathcal{D}$ is an expression of the form

$$
T(\mathbf{x}) \leftarrow R_{1}\left(\mathbf{y}_{1}\right), \ldots, R_{n}\left(\mathbf{y}_{n}\right),
$$

where every $R_{i}\left(\mathbf{y}_{i}\right)$ is an atom over $\mathcal{D}$, and $T(\mathbf{x})$ is an atom for which $T \notin \mathcal{D}$. Additionally, for safety, we require that every variable in $\mathbf{x}$ occurs in some $\mathbf{y}_{i}$. We refer to the head atom $T(\mathbf{x})$ by head ${ }_{\mathcal{Q}}$, and denote the set of body atoms $R_{i}\left(\mathbf{y}_{i}\right)$ by body $y_{\mathcal{Q}}$.

A conjunctive query is called full if all variables of the body also occur in the head. We say that a CQ is without self-joins when all of its atoms have a distinct relation name.

We denote by $\operatorname{vars}(\mathcal{Q})$ the set of all variables occurring in $\mathcal{Q}$. A valuation for a conjunctive query is a total function $V: \operatorname{vars}(\mathcal{Q}) \rightarrow$ dom that maps each variable of $\mathcal{Q}$ to a data value. We say that $V$ requires or needs the facts $V\left(\operatorname{body}_{\mathcal{Q}}\right)$ for $\mathcal{Q}$. A valuation $V$ is said to be satisfying for $\mathcal{Q}$ on instance $I$, when all the facts required by $V$ for $\mathcal{Q}$ are in $I$. In that case, $V$ derives the fact $V\left(\right.$ head $\left._{\mathcal{Q}}\right)$. The result of $\mathcal{Q}$ on instance $I$, denoted $\mathcal{Q}(I)$, is defined as the set of facts that can be derived by satisfying valuations for $\mathcal{Q}$ on $I$. We note that, as we do not allow negation, all conjunctive queries are monotone.

We frequently compare different valuations for a query $\mathcal{Q}$ with respect to their required sets of facts. For two valuations $V_{1}, V_{2}$ for a $\mathrm{CQ} \mathcal{Q}$, we write $V_{1} \leq_{Q} \quad V_{2}$ if $V_{1}\left(\right.$ head $\left._{\mathcal{Q}}\right)=V_{2}\left(\right.$ head $\left._{\mathcal{Q}}\right)$ and $V_{1}\left(\operatorname{bod} y_{\mathcal{Q}}\right) \subseteq V_{2}\left(\operatorname{bod} y_{\mathcal{Q}}\right)$. We write $V_{1}<_{Q} V_{2}$ if furthermore $V_{1}\left(\operatorname{bod}_{\mathcal{Q}}\right) \subsetneq V_{2}\left(\operatorname{bod}_{\mathcal{Q}}\right)$ holds.

A substitution is a mapping from variables to variables, which is generalized to tuples, atoms and conjunctive queries in the natural fashion [2] 2] We denote the composition of functions in the usual way, i.e., $(f \circ g)(x) \stackrel{\text { def }}{=} f(g(x))$.

The following notion is fundamental for the development in the rest of the paper:

Definition 2.1. A simplification of a conjunctive

\footnotetext{
${ }^{2}$ As we only consider CQs without constants, substitutions do not map variables to constants.
}

query $\mathcal{Q}$ is a substitution $\theta: \operatorname{vars}(\mathcal{Q}) \rightarrow \operatorname{vars}(\mathcal{Q})$ for which $\operatorname{head}_{\theta(\mathcal{Q})}=\operatorname{head}_{\mathcal{Q}}$ and $\operatorname{bod}_{\theta(\mathcal{Q})} \subseteq \operatorname{body}_{\mathcal{Q}}$.

A simplification is thus a homomorphism from $\mathcal{Q}$ to $\mathcal{Q}$ and by the homomorphism theorem [2] (and the trivial embedding from $\theta(\mathcal{Q})$ to $\mathcal{Q}), \mathcal{Q}$ and $\theta(\mathcal{Q})$ are equivalent. Of course, the identity substitution is always a simplification.

Example 2.2. We give a few examples to illustrate simplifications. Consider the query

$$
T(x) \leftarrow R(x, x), R(x, y), R(x, z) .
$$

Then $\theta_{1}=\{x \mapsto x, y \mapsto y, z \mapsto y\}$ as well as $\theta_{2}=$ $\{x \mapsto x, y \mapsto x, z \mapsto x\}$ are simplifications. For the query

$$
T(x) \leftarrow R(x, y), R(y, y), R(z, z), R(u, u),
$$

possible simplifications are $\theta_{3}=\{x \mapsto x, y \mapsto y, z \mapsto$ $y, u \mapsto z\}$ and $\theta_{4}=\{x \mapsto x, y \mapsto y, z \mapsto y, u \mapsto y\}$. For the query $T(x) \leftarrow R(x, y), R(y, z)$ there are no simplifications besides the identity.

The notion of simplification is closely related to foldings as defined by Chandra and Merlin [8]. In particular, a folding of a conjunctive query $\mathcal{Q}$ is a simplification $\theta$ that is idempotent. That is, $\theta^{2}=\theta$. Intuitively, the idempotence means that when $\theta$ gives a new name to a variable then it sticks to it. Notice that in Example 2.2 simplifications $\theta_{1}, \theta_{2}, \theta_{4}$ are foldings but $\theta_{3}$ is not as $\theta_{3}(u)=z \neq y=\theta_{3}\left(\theta_{3}(u)\right)$.

Networks, data distribution, and policies. A network $\mathcal{N}$ is a nonempty finite set of values from dom, which we call (computing) nodes.

A distribution policy $\boldsymbol{P}$ for a database schema $\mathcal{D}$ and a network $\mathcal{N}$ is a total function mapping facts from $\operatorname{facts}(\mathcal{D})$ to sets of nodes 3 For an instance $I$ over $\mathcal{D}$, let $\operatorname{dist}_{\boldsymbol{P}}(I)$ denote the function that maps each $\kappa \in \mathcal{N}$ to $\{\boldsymbol{f} \in I \mid \kappa \in \boldsymbol{P}(\boldsymbol{f})\}$, that is, the set of facts assigned to it by $\boldsymbol{P}$. We sometimes refer to $\operatorname{dist}_{\boldsymbol{P}}(I)(\kappa)$ as a data chunk.

\footnotetext{
${ }^{3}$ Notice that our formalization allows to 'skip' facts by mapping them to the empty set of nodes. This is, for instance, the case for a Hypercube distribution (cf. Section [5), which skips facts that are not essential to evaluate the query at hand.
} 
In this paper, we do not always explicitly give names to schemas and networks but tacitly assume they are understood from the queries and the distribution policies under consideration, respectively.

We do not always expect that distribution policies $\boldsymbol{P}$ are given as part of the input by exhaustive enumeration of all pairs $(\kappa, \boldsymbol{f})$, for which $\kappa \in \boldsymbol{P}(\boldsymbol{f})$. We also consider mechanisms, where instead the distribution policy is implicitly represented by a given "black box" procedure. While there are many possible ways to represent distribution policies, either as functions or as relations belonging to various complexity classes, in this paper, we only consider one such class. In particular, we define the class $\mathcal{P}_{\text {nrel }}$ where each distribution $\boldsymbol{P}$ is represented by a NPtestable relation, that on input $(\kappa, \boldsymbol{f})$ yields "true" if and only if $\kappa \in \boldsymbol{P}(\boldsymbol{f})$. We will discuss declarative ways to specify distribution policies in a non-blackbox fashion in Section [5]

The definition of a distribution policy is borrowed from Ameloot et al. 5] (but already surfaces in the work of Zinn et al. [17]), where distribution policies are used to define the class of policy-aware transducer networks.

\section{Parallel-Correctness}

In this section, we introduce and study the notion of parallel-correctness, which is central to this paper.

Definition 3.1. A query $\mathcal{Q}$ is parallel-correct on instance $I$ under distribution policy $\boldsymbol{P}$, if $\mathcal{Q}(I)=$ $\bigcup_{\kappa \in \mathcal{N}} \mathcal{Q}\left(\operatorname{dist}_{\boldsymbol{P}}(I)(\kappa)\right)$.

That is, the centralized execution of $\mathcal{Q}$ on $I$ is the same as taking the union of the results obtained by executing $\mathcal{Q}$ at every computing node. Next, we lift parallel-correctness to all instances.

Definition 3.2. A query $\mathcal{Q}$ is parallel-correct under distribution policy $\boldsymbol{P}$, if $\mathcal{Q}$ is parallel-correct on all input instances under $\boldsymbol{P}$.

Of course, when a query $\mathcal{Q}$ is parallel-correct under $\boldsymbol{P}$, there is a direct one-round evaluation algorithm for every instance. Indeed, the algorithm first distributes (reshuffles) the data over the computing nodes according to $\boldsymbol{P}$ and then evaluates $Q$ in a subsequent parallel step at every computing node. Notice that as $\boldsymbol{P}$ is defined on the granularity of a fact, the reshuffling does not depend on the current distribution of the data and can be done in parallel as well.

Although Definitions 3.1 and 3.2 are in terms of general queries, in the rest of this section, we only consider conjunctive queries. It is easy to see that a $\mathrm{CQ} \mathcal{Q}$ is parallel-correct under distribution policy $\boldsymbol{P}$ if for each valuation for $\mathcal{Q}$ the required facts meet at some node, i.e., if the following condition holds:

(C0) for every valuation $V$ for $\mathcal{Q}$,

$$
\bigcap_{\boldsymbol{f} \in V\left(\operatorname{bod} y_{\mathcal{Q}}\right)} \boldsymbol{P}(\boldsymbol{f}) \neq \emptyset .
$$

Even though $(\mathrm{C} 0)$ is sufficient for parallelcorrectness, it is not necessary (c.f., Example 3.5). It turns out that for a semantical characterization only valuations have to be considered that are minimal in the following sense.

Definition 3.3. Let $\mathcal{Q}$ be a CQ. A valuation $V$ for $\mathcal{Q}$ is minimal for $\mathcal{Q}$ if there exists no valuation $V^{\prime}$ for $\mathcal{Q}$ such that $V^{\prime}<_{\mathcal{Q}} V$.

The next lemma now states the targeted characterization:

Lemma 3.4. A $\mathrm{CQ} \mathcal{Q}$ is parallel-correct under distribution policy $\boldsymbol{P}$ if and only if the following holds:

(C1) for every minimal valuation $V$ for $\mathcal{Q}$,

$$
\bigcap_{\boldsymbol{f} \in V\left(\operatorname{bod} y_{\mathcal{Q}}\right)} \boldsymbol{P}(\boldsymbol{f}) \neq \emptyset .
$$

Proof (sketch). (if) Assume (C1) holds. Because of monotonicity, we only need to show that $\mathcal{Q}(I) \subseteq$ $\bigcup_{\kappa \in \mathcal{N}} \mathcal{Q}\left(\operatorname{dist}_{\boldsymbol{P}}(I)(\kappa)\right)$ for every instance $I$. To this end, let $\boldsymbol{f}$ be a fact that is derived by some valuation $V$ for $\mathcal{Q}$ over $I$. Then, there is also a minimal valuation $V^{\prime}$ that is satisfying on $I$ and which derives $\boldsymbol{f}$. Because of $(\mathrm{C} 1)$, there is a node $\kappa$ where all facts required for $V^{\prime}$ meet. Hence, $f \in$ $\bigcup_{\kappa \in \mathcal{N}} \mathcal{Q}\left(\operatorname{dist}_{\boldsymbol{P}}(I)(\kappa)\right)$. 
(only-if) Proof by contraposition. Suppose that there is a minimal valuation $V^{\prime}$ for $\mathcal{Q}$ for which the required facts do not meet under $\boldsymbol{P}$. Consider $V^{\prime}\left(\operatorname{bod}_{\mathcal{Q}}\right)$ as input instance. Then, by definition of minimality, there is no valuation that agrees on the head-variables and is satisfied on one of the chunks of $V^{\prime}\left(\operatorname{bod}_{\mathcal{Q}}\right)$ under $\boldsymbol{P}$. So, $\mathcal{Q}$ is not parallel-correct.

Example 3.5. For a simple example of a minimal valuation and a non-minimal valuation, consider the CQ $\mathcal{Q}$,

$$
T(x, z) \leftarrow R(x, y), R(y, z), R(x, x) .
$$

Both $V=\{x \mapsto a, y \mapsto b, z \mapsto a\}$ and $V^{\prime}=\{x \mapsto$ $a, y \mapsto a, z \mapsto a\}$ are valuations for $\mathcal{Q}$. Notice that both valuations agree on the head-variables of $\mathcal{Q}$, but they require different sets of facts. In particular, for $V$ to be satisfying on $I$, instance $I$ must contain the facts $R(a, b), R(b, a)$, and $R(a, a)$, while $V^{\prime}$ only requires $I$ to contain $R(a, a)$. This observation implies that $V$ is not minimal for $\mathcal{Q}$. Further, as $V^{\prime}$ requires only one fact for $\mathcal{Q}, V^{\prime}$ must be minimal for $\mathcal{Q}$.

We next argue that $(\mathrm{C} 0)$ is not a necessary condition for parallel-correctness. Indeed, take $\mathcal{N}=\{1,2\}$ and $\boldsymbol{P}$ as the distribution policy mapping every fact except $R(a, b)$ onto node 1 and every fact except $R(b, a)$ onto node 2 . Consider the valuations $V$ and $W=\{x \mapsto b, y \mapsto a, z \mapsto b\}$. Then, $R(a, b)$ and $R(b, a)$ do not meet under $\boldsymbol{P}$, thus violating condition (C0). It remains to argue that $\mathcal{Q}$ is parallel-correct under $\boldsymbol{P}$. For every minimal valuation $U$, either $\bigcap_{\boldsymbol{f} \in U\left(\operatorname{body}_{\mathcal{Q}}\right)} \boldsymbol{P}(\boldsymbol{f}) \neq \emptyset$ or $U$ requires both $R(a, b)$ and $R(b, a)$. But in the latter case $U$ is either valuation $V$ or $W$ as defined above which are not minimal. Thus, by Lemma 3.4 query $\mathcal{Q}$ is parallel-correct under $\boldsymbol{P}$.

Unfortunately, condition ( $\mathrm{C} 1$ ) is complexity-wise more involved than $(\mathrm{C} 0)$ as minimality of $V$ needs to be tested. The lower bound in Theorem 3.8 below indicates that this can, in a sense, not be avoided.

Towards an upper bound for the complexity of parallel-correctness, we first discuss how minimality of a valuation can be tested. Obviously, this notion is related to the (classical) notion of minimality for conjunctive queries, as we will make precise next. First, recall that a $\mathrm{CQ} \mathcal{Q}$ is minimal if there is no equivalent CQ with strictly less atoms.

Lemma 3.6. Let $\mathcal{Q}$ be a conjunctive query. For every injective valuation $V$ for $\mathcal{Q}$, it holds that $V$ is minimal if and only if $\mathcal{Q}$ is minimal 4

Lemma 3.6 immediately yields the following complexity result.

Proposition 3.7. Deciding whether a valuation $V$ for a $\mathrm{CQ} \mathcal{Q}$ is minimal is CONP-complete.

Proof (sketch). Lemma 3.6 yields a reduction from minimality of conjunctive queries to minimality of valuations. Therefore, coNP-hardness follows from the CONP-hardness of minimality for CQs, which follows from [12. The upper bound is immediate from the definition of minimality of valuations and from the fact that, for given $V_{1}, V_{2}, \mathcal{Q}$, it can be tested in polynomial time whether $V_{1}<_{\mathcal{Q}} V_{2}$ holds.

Now, we are ready to settle the complexity of parallel-correctness for general conjunctive queries for a large class of distributions. We study two settings, $\mathcal{P}_{\text {fin }}$, where distribution policies are explicitly enumerated as part of the input, and $\mathcal{P}_{\text {nrel }}$, where the distribution policy is given by a black box procedure which answers questions of the form " $\kappa \in \boldsymbol{P}(\boldsymbol{f})$ ?" in NP. In the latter case, the distribution is not part of the (normal) input and therefore does not contribute to the input size. Instead, the input has an additional parameter $n$ which bounds the length of addresses in the considered networks.

By $\mathbf{d o m}_{n}$ we denote the set of all elements of dom that can be encoded by strings of length at most $n$. For a distribution policy $\boldsymbol{P}$ (coming with a network $\mathcal{N}$ ) and a number $n$, we denote by $\boldsymbol{P}_{n}$ the distribution policy that is obtained from $\boldsymbol{P}$ by (1) only distributing facts over $\mathbf{d o m}_{n}$ and (2) only distributing facts to nodes whose addresses are of length at most $n$.

\footnotetext{
${ }^{4}$ We note that in a previous version of this paper, this lemma had a second statement (basically an explicit reduction from valuation minimality to CQ minimality), which turned out to be wrong. However, this second statement is not essential for the other results of the paper. Furthermore, from Proposition 3.7 it follows that a polynomial reduction from valuation minimality to $\mathrm{CQ}$ minimality indeed exists.
} 
We study the following algorithmic problems for explicitly given database instances:

$\operatorname{PCI}\left(\mathcal{P}_{\text {fin }}\right)$ :

Input: $\mathrm{CQ} \mathcal{Q}$, instance $I$, and $\boldsymbol{P} \in \mathcal{P}_{\text {fin }}$

Question: Is $\mathcal{Q}$ parallel-correct on $I$ under $\boldsymbol{P}$ ?

$\operatorname{PCI}\left(\mathcal{P}_{\text {nrel }}\right)$ :

Input: $\mathrm{CQ} \mathcal{Q}$, instance $I$, a natural number $n$ in unary representation

Black box input: $\boldsymbol{P} \in \mathcal{P}_{\text {nrel }}$

Question: Is $\mathcal{Q}$ parallel-correct on $I$ under $\boldsymbol{P}_{n}$ ?

We also study the parallel correctness problem without reference to a given database instance.

$\mathrm{PC}\left(\mathcal{P}_{\text {fin }}\right)$ :

Input: $\mathrm{CQ} \mathcal{Q}, \boldsymbol{P} \in \mathcal{P}_{\text {fin }}$

Question: Is $\mathcal{Q}$ parallel-correct on $I$ under $\boldsymbol{P}$, for all instances $I \subseteq \operatorname{facts}(\boldsymbol{P})$ ?

Here, $\operatorname{facts}(\boldsymbol{P})$ denotes the set of facts $\boldsymbol{f}$ with $\boldsymbol{P}(\boldsymbol{f}) \neq \emptyset$.

$\mathrm{PC}\left(\mathcal{P}_{\text {nrel }}\right)$ :

Input: $\mathrm{CQ} \mathcal{Q}$, a natural number $n$ in unary representation

Black box input: $\boldsymbol{P} \in \mathcal{P}_{\text {nrel }}$

Question: Is $\mathcal{Q}$ parallel-correct on $I$ under $\boldsymbol{P}_{n}$, for all instances $I \subseteq \operatorname{facts}\left(\boldsymbol{P}_{n}\right)$ ?

\section{Theorem 3.8.}

(a) $\operatorname{PC}\left(\mathcal{P}_{\text {fin }}\right)$ and $\operatorname{PCI}\left(\mathcal{P}_{\text {fin }}\right)$ are $\Pi_{2}^{P}$-complete.

(b) $\operatorname{PC}\left(\mathcal{P}_{\text {nrel }}\right)$ and $\operatorname{PCI}\left(\mathcal{P}_{\text {nrel }}\right)$ are in $\Pi_{2}^{P}$.

Proof (sketch). The upper bounds follow quite directly from Definition 3.1] or Lemma 3.4 and Proposition 3.7. respectively. The lower bound is by a reduction from the $\Pi_{2}^{P}$-complete $\Pi_{2}$-QBF-problem and uses a distribution policy with only two nodes. A proof is given in Appendix B.2.

Of course, the upper bounds in Theorem 3.8 also hold if questions of the form " $\kappa \in \boldsymbol{P}(\boldsymbol{f})$ ?" are answered in polynomial time, or if $\boldsymbol{P}$ is just given as a polynomial time function.
Due to the implicit representation of distributions, we cannot formally claim $\Pi_{2}^{p}$-hardness for distribution policies from $\mathcal{P}_{\text {nrel }}$. However, in an informal sense, they are, of course, at least as difficult as for $\mathcal{P}_{\text {fin }}$.

\section{Transferability}

While parallel-correctness provides a direct oneround evaluation algorithm, it still requires a reshuffling of the data for every query. It therefore makes sense, in the context of multiple query evaluation, to consider scenarios in which such reshuffling can be avoided. To this end, we introduce the notion of parallel-correctness transfer which ensures that a subsequent query $\mathcal{Q}^{\prime}$ can always be evaluated over a distribution for which a query $Q$ is parallel-correct:

Definition 4.1. For two queries $\mathcal{Q}$ and $\mathcal{Q}^{\prime}$ over the same input and output schema, parallel-correctness transfers from $\mathcal{Q}$ to $\mathcal{Q}^{\prime}$ when $\mathcal{Q}^{\prime}$ is parallel-correct under every distribution policy for which $\mathcal{Q}$ is parallelcorrect.

As for parallel-correctness we first give a semantical characterization before we study the complexity of parallel-correctness transfer. The proof of the following lemma is given in Appendix C.1

Lemma 4.2. Parallel-correctness transfers from a conjunctive query $\mathcal{Q}$ to a conjunctive query $\mathcal{Q}^{\prime}$ if and only if the following holds:

(C2) for every minimal valuation $V^{\prime}$ for $\mathcal{Q}^{\prime}$, there is a minimal valuation $V$ for $\mathcal{Q}$ such that $V^{\prime}\left(\operatorname{bod}_{\mathcal{Q}^{\prime}}\right) \subseteq V\left(\operatorname{bod}_{\mathcal{Q}}\right)$

Proof (sketch). (if) Let $\boldsymbol{P}$ be a distribution policy under which $\mathcal{Q}$ is parallel-correct. Let $I$ be an instance and $\boldsymbol{f}$ a fact in $\mathcal{Q}^{\prime}(I)$. It suffices to show that there is some valuation $V^{\prime}$ for $\mathcal{Q}^{\prime}$ that produces $\boldsymbol{f}$ at some node. Let $V^{\prime}$ be any minimal valuation that yields $\boldsymbol{f}$ and let $V$ be the minimal valuation of $\mathcal{Q}$, guaranteed by $(\mathrm{C} 2)$. Since $\mathcal{Q}$ is parallel-correct under $\boldsymbol{P}$ and $V$ is minimal for $\mathcal{Q}$, it follows by (C1) that all facts from $V\left(\operatorname{body}_{\mathcal{Q}}\right)$ must meet at some node $\kappa$. Since $V^{\prime}\left(\operatorname{bod} y_{\mathcal{Q}^{\prime}}\right) \subseteq V\left(\operatorname{bod}_{\mathcal{Q}}\right)$, all facts from 
$V^{\prime}\left(\right.$ body $\left._{\mathcal{Q}^{\prime}}\right)$ are available at node $\kappa$ and thus $\boldsymbol{f}$ is produced.

(only-if) Towards a contradiction, we assume that there is a minimal valuation $V^{\prime}$ for $\mathcal{Q}^{\prime}$, for which there is no valuation $V$ for $\mathcal{Q}$, where $V^{\prime}\left(\right.$ body $\left._{\mathcal{Q}^{\prime}}\right) \subseteq$ $V\left(\operatorname{bod}_{\mathcal{Q}}\right)$. Let $m=\left|V^{\prime}\left(\operatorname{body}_{\mathcal{Q}^{\prime}}\right)\right|$. In this sketch we only consider the case $m \geq 2$. Let $I \stackrel{\text { def }}{=} V^{\prime}\left(b_{0} d y_{\mathcal{Q}^{\prime}}\right)=$ $\left\{\boldsymbol{f}_{1}, \ldots, \boldsymbol{f}_{m}\right\}, \mathcal{N} \stackrel{\text { def }}{=}\left\{\kappa_{1}, \ldots, \kappa_{m}\right\}$, and let $\boldsymbol{P}$ be the distribution policy defined by

- $\boldsymbol{P}(\boldsymbol{g}) \stackrel{\text { def }}{=} \mathcal{N}$, for every $\boldsymbol{g} \in \operatorname{facts}(\mathcal{D}) \backslash I$; and

- $\boldsymbol{P}\left(\boldsymbol{f}_{i}\right) \stackrel{\text { def }}{=} \mathcal{N} \backslash\left\{\kappa_{i}\right\}$, for every $i$.

Intuitively, on every instance $J$, either the facts in $J$ meet on some node under $\boldsymbol{P}$, or $I \subseteq J$. By assumption, none of the minimal valuations for $\mathcal{Q}$ requires all the facts in $I$, implying that $\mathcal{Q}$ is parallel-correct under $\boldsymbol{P}$. Nevertheless, on instance $I$ under $\boldsymbol{P}$, none of the nodes receives all the facts in $I$, and (by minimality of $V^{\prime}$ ) there is no valuation that can derive $V^{\prime}\left(\right.$ head $\left._{\mathcal{Q}^{\prime}}\right)$ for a strict subset of the facts in $I$. So, $\mathcal{Q}^{\prime}$ is not parallel-correct under $\boldsymbol{P}$, which leads to the desired contradiction.

The above characterization allows us to pinpoint the complexity of parallel-correctness transfers. In particular, we consider the following problem:

PC-TRANS:

Input: CQs $\mathcal{Q}$ and $\mathcal{Q}^{\prime}$

Question: Does parallel-correctness transfer from $\mathcal{Q}$ to $\mathcal{Q}^{\prime}$ ?

Theorem 4.3. PC-TRANS is $\Pi_{3}^{P}$-complete.

Proof (idea). The upper bound again follows quite directly from the semantical characterization (Lemma 4.2) and Proposition 3.7. The lower bound is by a reduction from the $\Pi_{3}^{P}$-complete $\Pi_{3}$-QBF-problem. Let thus $\varphi=\forall \mathbf{x} \exists \mathbf{y} \forall \mathbf{z} \psi(\mathbf{x}, \mathbf{y}, \mathbf{z})$ be a formula with a quantifier-free propositional formula $\psi$ in 3 -DNF over variables $\mathbf{x}=\left(x_{1}, \ldots, x_{m}\right), \mathbf{y}=\left(y_{1}, \ldots, y_{n}\right)$, and $\mathbf{z}=\left(z_{1}, \ldots, z_{p}\right)$. From $\varphi$ we construct a CQ $\mathcal{Q}^{\prime}$ with head $H\left(x_{1}, \ldots, x_{m}, w_{1}, w_{0}\right)$ and a CQ $\mathcal{Q}$ with head $H\left(x_{1}, \ldots, x_{m}, y_{1}, \ldots, y_{n}, w_{1}, w_{0}\right)$. We then show, in a nutshell, that falseness of $\varphi$ corresponds to the existence of a valuation $V^{\prime}$ for $\mathcal{Q}^{\prime}$ (inducing a truth assignment $\beta_{\mathbf{x}}$ to $\mathbf{x}$ ), such that for every valuation $V$ for $\mathcal{Q}$ (with induced truth assignment $\left.\beta_{\mathbf{y}}\right)$, with $V^{\prime}\left(\operatorname{bod}_{\mathcal{Q}^{\prime}}\right) \subseteq V\left(\operatorname{bod} y_{\mathcal{Q}}\right)$, from any truth assignment $\beta_{\mathbf{z}}$ with $\left(\beta_{\mathbf{x}} \cup \beta_{\mathbf{y}} \cup \beta_{\mathbf{z}}\right) \not \forall \psi$ a valuation $V^{*}$ is obtained with $V^{*}<_{\mathcal{Q}} V$, implying that $V$ is not minimal. As such a truth assignment $\beta_{\mathbf{z}}$ exists (after the choice of $\beta_{\mathbf{x}}$ and for arbitrary $\beta_{\mathbf{y}}$ ) if and only if $\varphi$ is false, it can be shown that (C3) holds if and only if $\varphi$ is true.

A complete proof is given in Appendix C.2.

It is an easy observation that, if we require each valuation of $\mathcal{Q}$ to be minimal, then condition $(\mathrm{C} 2)$ yields a better, $\Pi_{2}^{P}$, complexity bound. Surprisingly, in this case, we even get a complexity drop to NP, as will be shown in Theorem 4.7 below. We next introduce the notions needed for this result.

Definition 4.4. A conjunctive query $\mathcal{Q}$ is strongly minimal if all its valuations are minimal.

We give some examples illustrating this definition. In Lemma 4.8, we present a sufficient condition for CQs to be strongly minimal.

Example 4.5. For an example of a strongly minimal query, consider query $\mathcal{Q}_{1}$,

$$
T\left(x_{1}, x_{2}, x_{2}, x_{4}\right) \leftarrow R\left(x_{1}, x_{2}\right), R\left(x_{2}, x_{3}\right), R\left(x_{3}, x_{4}\right) .
$$

Notice that, by fullness of $\mathcal{Q}_{1}$, there are no two distinct valuations for $\mathcal{Q}_{1}$ that derive the same fact. Hence, every valuation of $\mathcal{Q}_{1}$ must indeed be minimal.

For another example, consider the query $\mathcal{Q}_{2}$,

$$
T() \leftarrow R_{1}\left(x_{1}, x_{2}\right), R_{2}\left(x_{2}, x_{3}\right), R_{3}\left(x_{3}, x_{4}\right) .
$$

As each atom in the body of $\mathcal{Q}_{2}$ has a different relation symbol, each valuation of $\mathcal{Q}_{2}$ yields exactly three different facts and therefore, each valuation is minimal.

It is easy to see that every strongly minimal CQ is also a minimal CQ, but the converse is not true as witnessed by the query of Example 3.5, which is minimal but not strongly minimal. 
The following lemma now provides a characterization of parallel-correctness transfer for strongly minimal queries.

Lemma 4.6. Let $\mathcal{Q}^{\prime}$ be a $\mathrm{CQ}$ and let $\mathcal{Q}$ be a strongly minimal CQ. Parallel-correctness transfers from $\mathcal{Q}$ to $\mathcal{Q}^{\prime}$ if and only if the following holds:

(C3) there is a simplification $\theta$ for $\mathcal{Q}^{\prime}$ and a substitution $\rho$ for $\mathcal{Q}$ such that $\operatorname{bod}_{\theta\left(\mathcal{Q}^{\prime}\right)} \subseteq \operatorname{bod} y_{\rho(\mathcal{Q})}$.

Proof. We show that, for strongly minimal $\mathcal{Q},(\mathrm{C} 2)$ and (C3) are equivalent.

We first show that (C3) implies (C2). It suffices to show that if (C3) holds then for every minimal valuation $V^{\prime}$ for $\mathcal{Q}^{\prime}$, there is a valuation $V$ for $\mathcal{Q}$ such that $V^{\prime}\left(\operatorname{bod}_{\mathcal{Q}^{\prime}}\right) \subseteq V\left(\right.$ bod $\left._{\mathcal{Q}}\right)$. By strong minimality of $Q$, we can then conclude that $V$ is actually minimal.

Let $V^{\prime}$ be a minimal valuation for $\mathcal{Q}^{\prime}$ and let $\theta$ and $\rho$ be as in (C3). As $\theta$ is a simplification, $\operatorname{head}_{\theta\left(\mathcal{Q}^{\prime}\right)}=$ head $_{\mathcal{Q}^{\prime}}$ and body $y_{\theta\left(\mathcal{Q}^{\prime}\right)} \subseteq$ body $_{\mathcal{Q}^{\prime}}$. Therefore $\left(V^{\prime} \circ \theta\right)$ is also a valuation for $\mathcal{Q}^{\prime}$ with $\left(V^{\prime} \circ \theta\right)\left(\operatorname{bod} y_{\mathcal{Q}^{\prime}}\right)=$ $V^{\prime}\left(\operatorname{bod}_{\theta\left(\mathcal{Q}^{\prime}\right)}\right) \subseteq V^{\prime}\left(\operatorname{bod}_{\mathcal{Q}^{\prime}}\right)$ and by minimality of $V^{\prime}$ the latter inclusion is actually an equality.

By $(\mathrm{C} 3), \operatorname{bod}_{\theta\left(\mathcal{Q}^{\prime}\right)} \subseteq \operatorname{body}_{\rho(\mathcal{Q})}$, therefore $V^{\prime}$ is a partial valuation for $\rho(\mathcal{Q})$. Let $V^{\prime \prime}$ be some arbitrarily chosen extension of $V^{\prime}$ that is a (total) valuation for $\rho(\mathcal{Q})$. Then,

$V^{\prime}\left(\operatorname{bod}_{\mathcal{Q}^{\prime}}\right)=V^{\prime}\left(\operatorname{body}_{\theta\left(\mathcal{Q}^{\prime}\right)}\right)=V^{\prime \prime}\left(\operatorname{body}_{\theta\left(\mathcal{Q}^{\prime}\right)}\right)$

$$
\subseteq V^{\prime \prime}\left(\operatorname{bod}_{\rho(\mathcal{Q})}\right)=\left(V^{\prime \prime} \circ \rho\right)\left(\operatorname{bod}_{\mathcal{Q}}\right) .
$$

Thus, $V \stackrel{\text { def }}{=} V^{\prime \prime} \circ \rho$ is the desired valuation for $\mathcal{Q}$.

We next show that (C2) implies (C3). Actually, this implication even holds without the assumption that $\mathcal{Q}$ is strongly minimal. Let us therefore assume that (C2) holds. We choose $\theta$ as an arbitrary simplification that minimizes $\mathcal{Q}^{\prime}$. Such a simplification can be found thanks to 8 . In particular, $\theta\left(\mathcal{Q}^{\prime}\right)$ is a minimal $\mathrm{CQ}$ that is equivalent to $\mathcal{Q}^{\prime}$.

Let $V^{\prime}$ be an injective valuation for $\mathcal{Q}^{\prime}$. We claim that $V^{\prime} \circ \theta$ is a minimal valuation for $\mathcal{Q}^{\prime}$. Towards a contradiction, let us assume that there is a valuation $V^{\prime \prime}$ such that $V^{\prime \prime}<_{\mathcal{Q}^{\prime}} V^{\prime} \circ \theta$. Since $\theta$ is the identity on the head variables, $V^{\prime}$ is injective, and $V^{\prime}$ and $V^{\prime \prime}$ agree on $h_{e a d_{\mathcal{Q}}}$, we can conclude that
$\left(\left(V^{\prime}\right)^{-1} \circ V^{\prime \prime}\right)\left(\right.$ head $\left._{\mathcal{Q}^{\prime}}\right)=$ head $_{\mathcal{Q}^{\prime}}$, thus $\left(V^{\prime}\right)^{-1} \circ V^{\prime \prime}$ is a homomorphism from $\mathcal{Q}$ to $\left(\left(V^{\prime}\right)^{-1} \circ V^{\prime \prime}\right)(\mathcal{Q})$. Furthermore, $\left(\left(V^{\prime}\right)^{-1} \circ V^{\prime \prime}\right)\left(\operatorname{bod} y_{\mathcal{Q}}\right) \subseteq \operatorname{body}_{\theta(\mathcal{Q})} \subseteq$ bod $y_{\mathcal{Q}}$, therefore the identity is a homomorphism from $\left(\left(V^{\prime}\right)^{-1} \circ V^{\prime \prime}\right)(\mathcal{Q})$ to $\mathcal{Q}$. Together, $\left(\left(V^{\prime}\right)^{-1} \circ\right.$ $\left.V^{\prime \prime}\right)(\mathcal{Q})$ is equivalent to $\mathcal{Q}$. Furthermore, $\left(\left(V^{\prime}\right)^{-1} \circ\right.$ $\left.V^{\prime \prime}\right)\left(\operatorname{bod}_{\mathcal{Q}}\right) \subsetneq\left(V^{\prime}\right)^{-1}\left(V^{\prime}\left(\operatorname{bod}_{\mathcal{Q}}\right)\right)=\operatorname{body}_{\theta(\mathcal{Q})}$, contradicting the minimality of $\theta$. We thus conclude that $V^{\prime} \circ \theta$ is indeed a minimal valuation for $\mathcal{Q}^{\prime}$.

By (C2), there exists a minimal valuation $V$ for $\mathcal{Q}$ such that $V^{\prime}\left(\operatorname{bod}_{\theta\left(\mathcal{Q}^{\prime}\right)}\right)=\left(V^{\prime} \circ \theta\right)\left(\operatorname{bod}_{\mathcal{Q}^{\prime}}\right) \subseteq$ $V\left(\operatorname{body}_{\mathcal{Q}}\right)$. Now, let $f$ be an extension of $\left(V^{\prime}\right)^{-1}$, which maps values that occur in $V\left(\right.$ body $\left.y_{\mathcal{Q}}\right)$ but not in $V^{\prime}\left(b_{0} d y_{\mathcal{Q}^{\prime}}\right)$ in an arbitrary fashion and let $\rho \stackrel{\text { def }}{=}(f \circ V)$. Then,

$$
\begin{aligned}
& \operatorname{body}_{\theta\left(\mathcal{Q}^{\prime}\right)}=\left(V^{\prime}\right)^{-1}\left(V^{\prime}\left(\operatorname{body}_{\theta\left(\mathcal{Q}^{\prime}\right)}\right)\right) \\
& =f\left(V^{\prime}\left(\operatorname{bod}_{\theta\left(\mathcal{Q}^{\prime}\right)}\right)\right)=f\left(\left(V^{\prime} \circ \theta\right)\left(\operatorname{body}_{\mathcal{Q}^{\prime}}\right)\right) \\
& \subseteq f\left(V\left(\operatorname{bod}_{\mathcal{Q}}\right)\right)=\rho\left(\operatorname{bod} y_{\mathcal{Q}}\right)=\operatorname{bod} y_{\rho(\mathcal{Q})} .
\end{aligned}
$$

Thus, $\theta$ and $\rho$ witness condition (C3).

Theorem 4.7. PC-TRANS restricted to inputs with strongly minimal $\mathcal{Q}$ is NP-complete.

Proof (sketch). The upper bound follows from Lemma 4.6 by the observation that condition (C3) can be checked by a straighforward NP-algorithm. The lower bound follows from Proposition 5.4 below.

Theorem 4.7 assumes that it is known that $Q$ is strongly minimal. We complete the picture by investigating the complexity of the problem to decide whether a $\mathrm{CQ}$ is strongly minimal.

We first give a lemma that generalizes the above examples into a sufficient (but not necessary) condition for strong minimality.

In particular, Lemma 4.8 implies that every full CQ and every CQ without self-joins is strongly minimal. We say that an atom in a CQ is a self-join atom when the relation name of that atom occurs more than once in $\mathcal{Q}$. For instance, in the query $T() \leftarrow$ $R\left(x_{1}, x_{2}\right), R\left(x_{2}, x_{1}\right)$ both $R\left(x_{1}, x_{2}\right)$ and $R\left(x_{2}, x_{1}\right)$ are self-join atoms. 
Lemma 4.8. Let $\mathcal{Q}$ be a CQ. Then $\mathcal{Q}$ is strongly minimal when the following condition holds: if a variable $x$ occurs at a position $i$ in some self-join atom and not in the head of $\mathcal{Q}$, then all self-join atoms have $x$ at position $i$.

Proof (sketch). The proof is by contraposition, i.e., we show that if there is a valuation for $\mathcal{Q}$ which is not minimal then the condition is not satisfied. To this end, let $V$ and $V^{\prime}$ be valuations for $\mathcal{Q}$ which agree on the head-variables and where $V^{\prime}\left(\operatorname{bod} y_{\mathcal{Q}}\right) \subsetneq$ $V\left(\right.$ body $\left._{\mathcal{Q}}\right)$.

Then, there are at least two atoms $A_{1}=$ $R\left(x_{1}, \ldots, x_{k}\right)$ and $A_{2}=R\left(y_{1}, \ldots, y_{k}\right)$ in the body of $\mathcal{Q}$ that collapse under $V^{\prime}$, but not under $V$. That is, $V^{\prime}\left(A_{1}\right)=V^{\prime}\left(A_{2}\right)$ and $V\left(A_{1}\right) \neq V\left(A_{2}\right)$. So, under $V^{\prime}$ all the variables in $A_{1}$ and $A_{2}$ on matching positions must be mapped on the same constant, $V^{\prime}\left(x_{i}\right)=V^{\prime}\left(y_{i}\right)$ for each $i \in\{1, \ldots, k\}$, while for $V$ there is a position $j \in\{1, \ldots, k\}$ where this is not the case, $V\left(x_{j}\right) \neq V\left(y_{j}\right)$. Obviously, at least one of these variables must then be a non-head variable. So, either only $x_{j}$ is a head variable, or only $y_{j}$ is a head variable, or both are distinct non-head variables. In both cases the condition is not satisfied.

Example 4.9. For an example of a strongly minimal CQ that does not satisfy the condition in Lemma 4.8 . consider query $\mathcal{Q}_{3}$,

$$
T() \leftarrow R\left(x_{1}, x_{2}\right), R\left(x_{2}, x_{1}\right) .
$$

Notice that $\mathcal{Q}_{3}$ is indeed strongly minimal, because every valuation for $\mathcal{Q}_{3}$ either maps $x_{1}$ and $x_{2}$ on the same value, and thus requires only one fact where both values are equal, or maps $x_{1}$ and $x_{2}$ onto two distinct values, and thus requires exactly two facts where both values are distinct.

Finally, we establish the complexity of deciding strong minimality.

Lemma 4.10. Deciding whether a $\mathrm{CQ}$ is strongly minimal is coNP-complete.

Proof (sketch). The complement problem is easily seen to be in NP: for two guessed valuations $V^{*}, V$ (encoded in length polynomial of the query $\mathcal{Q}$ ) it can be checked in polynomial time whether $V^{*}<_{\mathcal{Q}} V$.

The lower bound is proved in Appendix C.3.

\section{Families of Distribution Poli- cies}

Parallel-correctness transfer can be seen as a generalization of parallel-correctness. In both cases, the goal is to decide whether a query can be correctly evaluated by evaluating it locally at each node. However, for parallel-correctness transfer, the question whether $\mathcal{Q}^{\prime}$ is parallel-correct is not asked for a particular distribution policy but for the family of those distribution policies, for which $\mathcal{Q}$ is parallel-correct 5

In this section, we study the parallel-correctness problem for other kinds of families of distribution policies that can be associated with a given query $\mathcal{Q}$. In Section [5.1, we will identify classes of families of policies, for which (C3) characterizes parallelcorrectness. For these classes we conclude that it is $\mathrm{NP}$-complete to decide, whether for the family $\mathcal{F}$ of policies associated with some given $\mathrm{CQ} \mathcal{Q}$, a $\mathrm{CQ} \mathcal{Q}^{\prime}$ is parallel-correct for all distributions from $\mathcal{F}$. In Section 5.2 we will see that this, in particular, holds for the families of distribution policies related to the practical Hypercube algorithm, that was previously investigated in several works [4, 6, 7, 10, 11. In fact, we even show that this holds for a more general class of distribution policies specified in a declarative formalism.

\subsection{Parallel-correctness}

We start with the following definition:

Definition 5.1. A query $\mathcal{Q}$ is parallel-correct for a family $\mathcal{F}$ of distribution policies if it is parallelcorrect under every distribution policy from $\mathcal{F}$.

We call a distribution policy $\boldsymbol{P} \mathcal{Q}$-generous for a $\mathrm{CQ} \mathcal{Q}$, if, for every valuation $V$ for $Q$, there is a node $\kappa$ that contains all facts from $V\left(\operatorname{bod} y_{\mathcal{Q}}\right)$. A family of distribution policies $\mathcal{F}$ is $\mathcal{Q}$-generous if every policy in $\mathcal{F}$ is. For an instance $I$, a distribution policy $\boldsymbol{P}$ is called $(\mathcal{Q}, I)$-scattered if for each node $\kappa$ there is a valuation $V$ for $\mathcal{Q}$, such that $\operatorname{dist}_{\boldsymbol{P}}(I)(\kappa) \subseteq V\left(\operatorname{body}_{\mathcal{Q}}\right)$. We then say that a family $\mathcal{F}$ of distribution policies

\footnotetext{
${ }^{5} \mathrm{~A}$ family of distribution policies is just a set of distribution policies.
} 
is $\mathcal{Q}$-scattered if $\mathcal{F}$ contains a $(\mathcal{Q}, I)$-scattered policy for every $I$. A $(\mathcal{Q}, I)$-scattered policy that is also $\mathcal{Q}$-generous yields the finest possible partition of the facts of $I$ and thus, intuitively, scatters them as much as possible.

Lemma 5.2. Let $\mathcal{Q}$ be a $\mathrm{CQ}$ and let $\mathcal{F}$ be a family of distribution policies that is $\mathcal{Q}$-generous and $\mathcal{Q}$ scattered. Then for every $\mathrm{CQ} \mathcal{Q}^{\prime}, \mathcal{Q}^{\prime}$ is parallel correct for $\mathcal{F}$ if and only if:

(C3) there is a simplification $\theta$ for $\mathcal{Q}^{\prime}$ and a substitution $\rho$ for $\mathcal{Q}$ such that $\operatorname{bod} y_{\theta\left(\mathcal{Q}^{\prime}\right)} \subseteq \operatorname{bod} y_{\rho(\mathcal{Q})}$.

We emphasize that Lemma 5.2 uses the same condition (C3) as Lemma 4.6.

Proof (sketch). (if) Let $I$ be a database for $\mathcal{Q}^{\prime}, \boldsymbol{P}$ a distribution policy from $\mathcal{F}$, and let $\theta$ and $\rho$ be as guaranteed by (C3). We show that each fact from $\mathcal{Q}^{\prime}(I)$ is produced at some node. Let $V^{\prime}$ be a valuation that yields some fact $\boldsymbol{h} \stackrel{\text { def }}{=} V^{\prime}\left(\right.$ head $\left._{\mathcal{Q}^{\prime}}\right)$ and let $V^{\prime \prime}$ be an arbitrary extension of $V^{\prime}$ for $\rho(\mathcal{Q})$. As $\theta$ is a simplification, $\left(V^{\prime} \circ \theta\right)$ also yields the fact $\boldsymbol{h}$. By (C3) we get $\left(V^{\prime} \circ \theta\right)\left(\operatorname{bod}_{\mathcal{Q}^{\prime}}\right)=V^{\prime}\left(\operatorname{bod} y_{\theta\left(\mathcal{Q}^{\prime}\right)}\right) \subseteq V^{\prime \prime}\left(\operatorname{bod} y_{\rho(\mathcal{Q})}\right)=$ $\left(V^{\prime \prime} \circ \rho\right)\left(\operatorname{body}_{\mathcal{Q}}\right)$. As $\boldsymbol{P}$ is $\mathcal{Q}$-generous, there is some node $\kappa$ that has all facts from $\left(V^{\prime \prime} \circ \rho\right)\left(\right.$ body $\left.y_{\mathcal{Q}}\right)$ and therefore all facts from $\left(V^{\prime} \circ \theta\right)\left(b_{o d y_{\mathcal{Q}^{\prime}}}\right)$, and thus $\boldsymbol{h}$ is produced at $\kappa$.

(only-if) Suppose $\mathcal{Q}^{\prime}$ is parallel-correct under all distribution policies in $\mathcal{F}$. Let $V^{\prime}$ be some injective

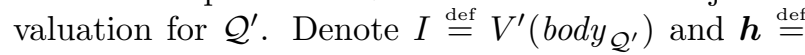
$V^{\prime}\left(\right.$ head $\left._{\mathcal{Q}^{\prime}}\right)$. Let $\boldsymbol{P}$ be some $(\mathcal{Q}, I)$-scattered distribution policy from $\mathcal{F}$. Because $\mathcal{Q}^{\prime}$ is parallel-correct under $\boldsymbol{P}$, there must be a node $\kappa$ that outputs $\boldsymbol{h}$ when $I$ is distributed according to $\boldsymbol{P}$. Therefore, there is a valuation $W^{\prime}$ for $\mathcal{Q}^{\prime}$ such that $\kappa$ contains all facts from $W^{\prime}\left(\right.$ body $\left._{\mathcal{Q}^{\prime}}\right)$ and $W^{\prime}\left(\right.$ head $\left._{\mathcal{Q}^{\prime}}\right)=\boldsymbol{h}$. We claim that $\theta \stackrel{\text { def }}{=}\left(V^{\prime}\right)^{-1} \circ W^{\prime}$ is a simplification of $\mathcal{Q}^{\prime}$. Indeed, this substitution is well-defined thanks to the injectivity of $V^{\prime}$ and furtermore $\left(\left(V^{\prime}\right)^{-1} \circ W^{\prime}\right)\left(\right.$ head $\left._{\mathcal{Q}^{\prime}}\right)=$ head $_{\mathcal{Q}^{\prime}}$ and $\left(\left(V^{\prime}\right)^{-1} \circ W^{\prime}\right)\left(\right.$ body $\left._{\mathcal{Q}^{\prime}}\right) \subseteq$ body $_{\mathcal{Q}^{\prime}}$, as $W^{\prime}\left(\operatorname{bod}_{\mathcal{Q}^{\prime}}\right) \subseteq I=V^{\prime}\left(\operatorname{body}_{\mathcal{Q}^{\prime}}\right)$ and $\left(\bar{V}^{\prime}\right)^{-1}$ maps $I$ back to body $y_{\mathcal{Q}^{\prime}}$.

As $\boldsymbol{P}$ is $(\mathcal{Q}, I)$-scattered, there is a valuation $V$ such that $\operatorname{dist}_{\boldsymbol{P}}(I)(\kappa) \subseteq V\left(\operatorname{body}_{\mathcal{Q}}\right)$. Then, let $g$ be some mapping from $i m g(V)$ to var such that for all $d \in i m g\left(W^{\prime}\right), g(d)=g^{\prime}(d)$. We define the renaming $\rho \stackrel{\text { def }}{=} g \circ V$ and show that with these choices, $\operatorname{body}_{\theta\left(\mathcal{Q}^{\prime}\right)} \subseteq \operatorname{bod}_{\rho(\mathcal{Q})}$, and thus (C3) holds.

Let $R\left(x_{1}, \ldots, x_{k}\right) \in \operatorname{bod} y_{\theta\left(\mathcal{Q}^{\prime}\right)}$. Then, there is an atom $R\left(y_{1}, \ldots, y_{k}\right) \in \operatorname{body}_{\mathcal{Q}^{\prime}}$ with $W^{\prime}(R(\bar{y})) \in$ $\operatorname{dist}_{\boldsymbol{P}}(I)(\kappa)$ and, for each $i, x_{i}=\left(V^{\prime}\right)^{-1}\left(W^{\prime}\left(y_{i}\right)\right)$. So, as $\operatorname{dist}_{\boldsymbol{P}}(I)(\kappa) \subseteq V\left(\operatorname{bod}_{\mathcal{Q}}\right), W^{\prime}(R(\bar{y})) \in V\left(\operatorname{bod}_{\mathcal{Q}}\right)$ and there is some atom $R\left(z_{1}, \ldots, z_{k}\right) \in \operatorname{body}_{\mathcal{Q}}$ such that $W^{\prime}(R(\bar{y}))=V(R(\bar{z}))$. Clearly, $W^{\prime}\left(y_{i}\right)=V\left(z_{i}\right)$ for all $i$. By definition of $g$, it then follows that $x_{i}=\left(V^{\prime}\right)^{-1}\left(W^{\prime}\left(y_{i}\right)\right)=g\left(V\left(z_{i}\right)\right)$ for all $i$. Therefore, $R\left(x_{1}, \ldots, x_{k}\right) \in \operatorname{body}_{\rho(\mathcal{Q})}$, as desired.

Theorem 5.3. It is NP-complete to decide, for given CQs $\mathcal{Q}$ and $\mathcal{Q}^{\prime}$, whether $\mathcal{Q}^{\prime}$ is parallel-correct for $\mathcal{Q}$-generous and $\mathcal{Q}$-scattered families of distribution policies.

The proof of this theorem shows in particular, that $\mathcal{Q}^{\prime}$ is either parallel-correct for all $\mathcal{Q}$-generous and $\mathcal{Q}$ scattered families of distribution policies or for none of them.

Proof (sketch). The upper bound follows from Lemma 5.2 and the fact that (C3) can be checked by an NP-algorithm. Indeed such an algorithm only needs to guess $\theta$ and $\rho$ and to verify (in polynomial time) that $\operatorname{bod}_{\theta\left(\mathcal{Q}^{\prime}\right)} \subseteq \operatorname{bod} y_{\rho(\mathcal{Q})}$.

The lower bound follows by Lemma 5.2 and the following Proposition 5.4.

Proposition 5.4. It is NP-hard to decide, whether for CQs $\mathcal{Q}$ and $\mathcal{Q}^{\prime}$ condition (C3) holds. This statement remains true if either $\mathcal{Q}$ or $\mathcal{Q}^{\prime}$ is restricted to acyclic queries. It also remains true if both CQs are Boolean and if $\mathcal{Q}$ is full.

Remark 5.5. The proof of Proposition [5.4 is given in Appendix D.1. In both cases $\left(\mathcal{Q}\right.$ acyclic or $\mathcal{Q}^{\prime}$ acyclic) it is by a reduction from graph 3-colorability. The first reduction, in which the input graph is encoded in $\mathcal{Q}^{\prime}$ and the valid color-assignments in $\mathcal{Q}$ is straightforward. As it only uses a fixed number of colors, $\mathcal{Q}$ can be made acyclic by adding an atom to $\mathcal{Q}$ that contains all allowed colors.

The second reduction, in which the graph is encoded in $\mathcal{Q}$ and the valid color-assignments in $\mathcal{Q}^{\prime}$, is a bit more involved. 
The reader may now wonder whether NP-hardness remains when both $\mathcal{Q}$ and $\mathcal{Q}^{\prime}$ are required to be acyclic. When relations of arbitrary arity are allowed, this is indeed the case: acyclicity is then easily achieved by using one atom containing all variables of the query. Under bounded-arity database schemas, however, the complexity of parallel-correctness transfer for acylic queries remains open.

\subsection{Hypercube Distribution Policies}

In the following, we give a short definition of $\mathrm{Hy}$ percube distributions and settle the complexity of the parallel-correctness transfer problem for families $\mathcal{H}(\mathcal{Q})$ of Hypercube distributions for some $\mathrm{CQ} \mathcal{Q}$ with the help of the results of Section 5.1. We highlight how Hypercube distributions can be specified in a rule-based fashion, which we consider useful also for more general distributions.

Let $\mathcal{Q}$ be a conjunctive query with (body) variables $x_{1}, \ldots, x_{k}$. A collection $H=\left(h_{1}, \ldots, h_{k}\right)$ of hash function: 6 (called a hypercube in the following) determines a hypercube distribution $\boldsymbol{P}_{H}$ for $\mathcal{Q}$ in the following way. For each $i \in\{1, \ldots, k\}$, we let $A_{i} \stackrel{\text { def }}{=} i m g\left(h_{i}\right)$ and define the address space $\mathcal{A}$ of $\boldsymbol{P}_{H}$ as the cartesian product $A_{1} \times \cdots \times A_{k}$.

In a nutshell, $\boldsymbol{P}_{H}$ has one node per address in $\mathcal{A}$ and distributes, for every valuation $V$ of $\mathcal{Q}$, every fact $\boldsymbol{f}=V(A)$, where $A$ is an atom of $\mathcal{Q}$, to all nodes whose address $\left(a_{1}, \ldots, a_{k}\right)$ satisfies $a_{i}=h_{i}\left(V\left(x_{i}\right)\right)$, for all variables $x_{i}$ occurring in $A$.

For the declarative specification of $\boldsymbol{P}_{H}$ we make use of predicates 7 bucket $i$ and bucket $_{i}^{*}$, where bucket $_{i}(a, b)$ holds, if $h_{i}(a)=b$, and bucket ${ }_{i}^{*}(b)$ holds, if $b \in i m g\left(h_{i}\right)$.

With these predicates, $\boldsymbol{P}_{H}$ can be specified by stating, for each atom $R\left(y_{1}, \ldots, y_{m}\right)$ of $\mathcal{Q}$, one rule

$$
\begin{aligned}
T_{R}\left(z_{1}, \ldots, z_{k} ; y_{1}, \ldots, y_{m}\right) \leftarrow & R\left(y_{1}, \ldots, y_{m}\right), \\
& B_{1}, \ldots, B_{k} .
\end{aligned}
$$

Here, for each $i \in\{1, \ldots, k\}, B_{i}$ is $\operatorname{bucket}_{i}\left(x_{i}, z_{i}\right)$, if

\footnotetext{
${ }^{6} \mathrm{~A}$ hash function is a partial mapping from dom to a finite set whose elements are sometimes referred to as buckets.

${ }^{7}$ For the purpose of specification it is irrelevant whether these predicates are materialized in the database.
}

$x_{i}$ occurs in $y_{1}, \ldots, y_{m}$, and $B_{i}$ is $\operatorname{bucket}_{i}^{*}\left(z_{i}\right)$, otherwise.

The semantics of such a rule is straightforward. For each valuation $V$ of the variables $z_{1}, \ldots, z_{k}, x_{1}, \ldots, x_{k}$, that makes the body of the rule true, the fact $R\left(V\left(y_{1}\right), \ldots, V\left(y_{m}\right)\right)$ is distributed to the node with address $\left(V\left(z_{1}\right), \ldots, V\left(z_{k}\right)\right)$. We emphasize that the variables $y_{1}, \ldots, y_{m}$ need not be pairwise distinct and that $\left\{y_{1}, \ldots, y_{m}\right\} \subseteq\left\{x_{1}, \ldots, x_{k}\right\}$.

Remark 5.6. It is evident that one could use more general rules to specify distribution policies. More than one atom with a database relation could be in the body, and there could be other additional predicates than those derived from hashing functions. Furthermore, the address space could be defined differently.

For a $\mathrm{CQ} \mathcal{Q}$, we denote by $\mathcal{H}_{\mathcal{Q}}$ the family of distribution policies $\left\{\boldsymbol{P}_{H} \mid H\right.$ is a hypercube for $\left.\mathcal{Q}\right\}$.

Lemma 5.7. Let $\mathcal{Q}$ be a CQ. Then $\mathcal{H}_{\mathcal{Q}}$ is $\mathcal{Q}$-generous and $\mathcal{Q}$-scattered.

Proof. Let $Q$ be a CQ with $\operatorname{vars}(\mathcal{Q})=\left\{u_{1}, \ldots, u_{k}\right\}$.

We first show that every policy $\boldsymbol{P}_{H} \in \mathcal{H}_{\mathcal{Q}}$ is $\mathcal{Q}$ generous. To this end, let $H$ be a hypercube and let $V$ be a valuation for $\mathcal{Q}$. Then, by definition, for the node $\kappa$ with address $\left(h_{1}\left(V\left(u_{1}\right)\right), \ldots, h_{k}\left(V\left(u_{k}\right)\right)\right)$, $\kappa \in \boldsymbol{P}_{H}(\boldsymbol{f})$ for every $\boldsymbol{f} \in V\left(\operatorname{bod}_{\mathcal{Q}}\right)$.

We now show that $\mathcal{H}_{\mathcal{Q}}$ is $\mathcal{Q}$-scattered. Thereto, let $I$ be an instance. For every $i \leq k$, we choose $A_{i} \stackrel{\text { def }}{=} \operatorname{adom}(I)$ and let $h_{i}(a) \stackrel{\text { def }}{=} a$, for every $a \in A_{i}$. Let $\kappa$ be an arbitrary node and let $\left(a_{1}, \ldots, a_{k}\right)$ be its address. Let $V$ be the valuation mapping $u_{i}$ to $a_{i}$, for each $i$. Let $R\left(d_{1}, \ldots, d_{m}\right) \in \operatorname{dist}_{\boldsymbol{P}_{H}}(I)(\kappa)$ thanks to some rule

$$
\begin{aligned}
T_{R}\left(z_{1}, \ldots, z_{k} ; y_{1}, \ldots, y_{m}\right) \leftarrow R\left(y_{1}, \ldots, y_{m}\right), \\
B_{1}, \ldots, B_{k} .
\end{aligned}
$$

By definition of the hash functions, every valuation that satisfies the body of this rule, maps $x_{i}$ to $a_{i}$, for every $x_{i}$ that appears in $R\left(y_{1}, \ldots, y_{m}\right)$. However, as this valuation coincides with $V$ on $y_{1}, \ldots, y_{m}$, it maps $R\left(y_{1}, \ldots, y_{m}\right)$ to an element of $V\left(\operatorname{bod} y_{\mathcal{Q}}\right)$. Therefore, $\operatorname{dist}_{\boldsymbol{P}_{H}}(I)(\kappa) \subseteq V\left(\operatorname{bod}_{\mathcal{Q}}\right)$. 
Corollary 5.8. It is NP-complete to decide, for given conjunctive queries $\mathcal{Q}, \mathcal{Q}^{\prime}$, whether $\mathcal{Q}^{\prime}$ is parallel-correct for $\mathcal{H}_{\mathcal{Q}}$.

Remark 5.9. It is easy to see that Lemma 5.7 and then the upper bound of Corollary 5.8 holds for more general families of distribution policies. As an example, one could add further atoms of $\mathcal{Q}$ as "filters" to the bodies of the above rules.

\section{Conclusions}

We have introduced parallel-correctness as a framework for studying one-round evaluation algorithms for the evaluation of queries under arbitrary distribution policies. We have obtained tight bounds on the complexity of deciding parallel-correctness and the transferability problem for conjunctive queries. For general conjunctive queries, these complexities reside in different levels of the polynomial hierarchy (even when considering Hypercube distributions). Since the considered problems are static analysis problems that relate to queries and not to instances (at least in the case of transferability), such complexities do not necessarily put a burden on practical applicability. Still, it would be interesting to identify fragments of conjunctive queries or particular classes of distribution policies that could render these problems tractable. In addition, it would be interesting to explore more expressive classes of queries and other families of distribution policies.

The notion of parallel-correctness is directly inspired by Hypercube where the result of the query is obtained by aggregating (through union) the evaluation of the original query over the distributed instance. Other possibilities are to consider more complex aggregator functions than union and to allow for a different query than the original one to be executed at computing nodes.

\section{Acknowledgments}

We thank Serge Abiteboul, Luc Segoufin, Cristina Sirangelo, and Thomas Zeume for helpful remarks.

\section{References}

[1] Spark. http://spark.apache.org.

[2] S. Abiteboul, R. Hull, and V. Vianu. Foundations of Databases. Addison-Wesley, 1995.

[3] F. N. Afrati, P. Koutris, D. Suciu, and J. D. Ullman. Parallel skyline queries. In Deutsch 9], pages 274284.

[4] F. N. Afrati and J. D. Ullman. Optimizing joins in a map-reduce environment. In EDBT, pages 99-110, 2010.

[5] T. J. Ameloot, B. Ketsman, F. Neven, and D. Zinn. Weaker forms of monotonicity for declarative networking: a more fine-grained answer to the CALMconjecture. In Hull and Grohe 13, pages 64-75.

[6] P. Beame, P. Koutris, and D. Suciu. Communication steps for parallel query processing. In $P O D S$, pages 273-284, 2013.

[7] P. Beame, P. Koutris, and D. Suciu. Skew in parallel query processing. In Hull and Grohe [13], pages 212223.

[8] A. K. Chandra and P. M. Merlin. Optimal implementation of conjunctive queries in relational data bases. In $S T O C$, pages 77-90, 1977.

[9] A. Deutsch, editor. 15th International Conference on Database Theory, ICDT '12, Berlin, Germany, March 26-29, 2012. ACM, 2012.

[10] S. Ganguly, A. Silberschatz, and S. Tsur. A framework for the parallel processing of Datalog queries. In SIGMOD, pages 143-152, 1990.

[11] S. Ganguly, A. Silberschatz, and S. Tsur. Parallel bottom-up processing of datalog queries. J. Log. Program., 14(1\&2):101-126, 1992.

[12] P. Hell and J. Nesetril. The core of a graph. Discrete Mathematics, 109(1-3):117-126, 1992.

[13] R. Hull and M. Grohe, editors. Proceedings of the 33rd ACM SIGMOD-SIGACT-SIGART Symposium on Principles of Database Systems, PODS'14, Snowbird, UT, USA, June 22-27, 2014. ACM, 2014.

[14] P. Koutris and D. Suciu. Parallel evaluation of conjunctive queries. In PODS, pages 223-234, 2011.

[15] L. J. Stockmeyer. The polynomial-time hierarchy. Theor. Comput. Sci., 3(1):1-22, 1976.

[16] R. Xin, J. Rosen, M. Zaharia, M. Franklin, S. Shenker, and I. Stoica. Shark: SQL and rich analytics at scale. In SIGMOD, 2013. 
[17] D. Zinn, T. J. Green, and B. Ludäscher. Win-move is coordination-free (sometimes). In Deutsch 9], pages 99-113. 


\section{Appendix}

\section{A Preliminaries}

We recapitulate some classical definitions and facts regarding the polynomial hierarchy, which will be used in the following proofs for lower and upper bounds. We are only concerned with classes $\operatorname{CoNP}=\Pi_{1}^{P}, \Pi_{2}^{P}$, and $\Pi_{3}^{P}$ of the first, second, and third level of the hierarchy as well as with their complement classes $\mathrm{NP}=\Sigma_{1}^{P}$, $\Sigma_{2}^{P}$, and $\Sigma_{3}^{P}$.

Definition A.1. Class $\Pi_{3}^{P}$ consists of those problems $L$ over an alphabet $\Sigma$, for which there is an algorithm $\mathcal{A}$ and a polynomial $p$ such that a string $w \in \Sigma^{*}$ is in $L$ if and only if for every $x \in \Sigma^{*}$ there exists $y \in \Sigma^{*}$ such that for every $z \in \Sigma^{*}$ algorithm $\mathcal{A}$ accepts $(w, x, y, z)$ and its running time is bounded by $p(|w|)$.

Similarly, $\Pi_{2}^{P}$ consists of those $L$, for which there is an algorithm $\mathcal{A}$ and a polynomial $p$ such that a string $w \in \Sigma^{*}$ is in $L$ if and only if for every $x \in \Sigma^{*}$ there exists $y \in \Sigma^{*}$ such that algorithm $\mathcal{A}$ accepts $(w, x, y)$ and its running time is bounded by $p(|w|)$.

Remark A.2. When we show that an algorithmic problem belongs to one of these classes, we do not apply the definition literally. Instead, as usual, we deal with mathematical objects like relations and valuations directly and assume that their encoding as strings is done in a straightforward way.

The following problem for quantified boolean formulæ $(\mathrm{QBF})$ is complete class $\Pi_{3}^{P}$. The quantifier structure of the input resembles that for inputs $x, y, z$ in the acceptance condition for the algorithm in Definition A.1.

Problem: $\Pi_{3}-\mathrm{QBF}$

Input: $\quad$ Formula $\varphi=\forall \mathbf{x} \exists \mathbf{y} \forall \mathbf{z} \psi(\mathbf{x}, \mathbf{y}, \mathbf{z})$ where $\psi$ is a propositional formula

Question: Does for every truth assignment $\beta_{\mathbf{x}}$ on $\mathbf{x}$ exist a truth assignment $\beta_{\mathbf{y}}$ on $\mathbf{y}$ such that for every truth assignment $\beta_{\mathbf{z}}$ on $\mathbf{z}$, truth assignment $\beta \stackrel{\text { def }}{=}\left(\beta_{\mathbf{x}} \cup \beta_{\mathbf{y}} \cup \beta_{\mathbf{z}}\right)$ satisfies $\psi, \beta \models \psi$ ?

Problem $\Pi_{2}-\mathrm{QBF}$ is defined analogously with formulas of the form $\varphi=\forall \mathbf{x} \exists \mathbf{y} \psi(\mathbf{x}, \mathbf{y})$. In general, the number $i$ of quantifier blocks in an input formula for $\Pi_{i}$-QBF corresponds with level $i$ of class $\Pi_{i}^{P}$ in the polynomial hierarchy. Also, the complement classes $\Sigma_{2}^{P}=\mathrm{CO}-\Pi_{2}^{P}$ and $\Sigma_{3}^{P}=\mathrm{CO}-\Pi_{3}^{P}$ are defined like $\Pi_{2}^{P}$ and $\Pi_{3}^{P}$, respectively, but every quantifier is replaced by its dual.

Remark A.3. Problems $\Pi_{2}-\mathrm{QBF}$ and $\Pi_{3}$-QBF are well-known to be complete for $\Pi_{2}^{P}$ and $\Pi_{3}^{P}$, respectively. They remain hard if $\psi$ is assumed to be in $3-\mathrm{CNF}$ (for $\Pi_{2}-\mathrm{QBF}$ ) or in 3-DNF (for $\left.\Pi_{3}-\mathrm{QBF}\right)$ [15, Theorem 4.1.2].

Below we often use the notation $\langle o\rangle$ for an object $o$ to denote some reasonable string encoding of $o$ over some finite alphabet.

\section{B Proofs for Section 3: Parallel-Correctness}

\section{B.1 Proof of Lemma 3.6}

In the following let $\mathcal{Q}$ be a $\mathrm{CQ}$.

We show that there is a non-minimal injective valuation $V$ for $\mathcal{Q}$ if and only if $\mathcal{Q}$ is not minimal. 
(if) Suppose that $\mathcal{Q}$ is not minimal. Then, by [8] there is a folding $h$ for $\mathcal{Q}$, where body $y_{h(\mathcal{Q})} \subsetneq$ body $y_{\mathcal{Q}}$ and $\operatorname{head}_{h(\mathcal{Q})}=$ head $_{\mathcal{Q}}$. Let $V$ be an arbitrary injective valuation for $\mathcal{Q}$. Injectivity implies that $\mid V\left(\right.$ bod $\left.y_{\mathcal{Q}}\right) \mid=$ $\mid$ body $y_{\mathcal{Q}} \mid$, that is the number of facts in $V\left(\right.$ body $\left.y_{\mathcal{Q}}\right)$ equals the number of atoms in $\mid$ body $y_{\mathcal{Q}} \mid \cdot$

Since $h(\mathcal{Q})$ only has variables that also appear in $\mathcal{Q}, V$ is a valuation for $h(\mathcal{Q})$ as well. However, thanks

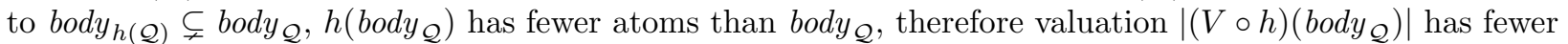
facts than $V\left(\right.$ bod $\left.y_{\mathcal{Q}}\right)$. Thus, $(V \circ h)$ is a counterexample for the minimality of $V$, since $(V \circ h)\left(b o d y_{\mathcal{Q}}\right)=$ $V\left(\operatorname{body}_{h(\mathcal{Q})}\right) \subseteq V\left(\right.$ body $\left._{\mathcal{Q}}\right)$ and $(V \circ h)\left(\right.$ head $\left._{\mathcal{Q}}\right)=V\left(\right.$ head $\left._{h(\mathcal{Q})}\right)=V\left(\right.$ head $\left._{\mathcal{Q}}\right)$

(only-if) Suppose there is an injective valuation $V$ for $\mathcal{Q}$ and a valuation $V^{\prime}$ for $\mathcal{Q}$, such that $V^{\prime}<_{\mathcal{Q}}$ $V$. Then, $h \stackrel{\text { def }}{=}\left(V^{-1} \circ V^{\prime}\right)$ is a homomorphism from $\mathcal{Q}$ to itself, as body $y_{(\mathcal{Q})}=V^{-1}\left(V^{\prime}\left(\operatorname{bod} y_{\mathcal{Q}}\right)\right) \subsetneq$ $V^{-1}\left(V\left(\operatorname{bod}_{\mathcal{Q}}\right)\right)=\operatorname{bod}_{\mathcal{Q}}$, and $h e a d_{h(\mathcal{Q})}=V^{-1}\left(V^{\prime}\left(\operatorname{head}_{\mathcal{Q}}\right)\right)=V^{-1}\left(V\left(\operatorname{head}_{\mathcal{Q}}\right)\right)=h_{e a d_{\mathcal{Q}}}$. Therefore $h(\mathcal{Q})$ equivalent to $\mathcal{Q}$, thanks to the homomorphism theorem (see, e.g., [2]).

\section{B.2 Proof of Theorem 3.8}

The upper bounds in Theorem 3.8(b) follow from Proposition B.2 for PCI $\left(\mathcal{P}_{\text {nrel }}\right)$ and Proposition B.5 for $\mathrm{PC}\left(\mathcal{P}_{\text {nrel }}\right)$. They also imply the upper bounds of Theorem 3.8 (a), as stated in Corollary B.3 for PCI $\left(\mathcal{P}_{\text {fin }}\right)$ and Corollary B.6 for $\mathrm{PC}\left(\mathcal{P}_{\text {fin }}\right)$. The matching lower bound for $\mathrm{PCI}\left(\mathcal{P}_{\text {fin }}\right)$ is stated in Proposition B.7 and the matching lower bound for $\mathrm{PC}\left(\mathcal{P}_{\text {fin }}\right)$ in Proposition B.8.

\section{B.2.1 Upper bounds}

Before we prove the upper bounds, we discuss how to use distribution policies from $\mathcal{P}_{\text {nrel }}$.

A distribution policy $\boldsymbol{P} \in \mathcal{P}_{\text {nrel }}$ is an NP-testable relation. This means there exists a (deterministic) algorithm $\mathcal{A}_{\boldsymbol{P}}$ with time bound a polynomial in $\langle\kappa, \boldsymbol{f}\rangle$ that accepts input $(\langle\kappa, \boldsymbol{f}\rangle, x)$ for some string $x$ if and only if $\kappa \in \boldsymbol{P}(\boldsymbol{f})$. We use algorithm $\mathcal{A}_{\boldsymbol{P}}$ as a subroutine in the following algorithms, as described below.

Remark B.1 (Use of subroutine $\mathcal{A}_{\boldsymbol{P}}$ ). Let $V$ be a valuation for a query $\mathcal{Q}$ with $k$ body atoms and let $\kappa$ be some node. We assume some additional input string $x=x_{1} \circ \cdots \circ x_{k}$, where each substring $x_{i}$ has a length polynomial in $V\left(b o d y_{\mathcal{Q}}\right)$ and the representation size of $\kappa$. An algorithm can "test" (w.r.t. $x$ ) whether there is a fact in $V\left(\operatorname{bod} y_{\mathcal{Q}}\right)=\left\{\boldsymbol{f}_{1}, \ldots, \boldsymbol{f}_{\ell}\right\}$ that is not assigned to node $\kappa$ under distribution policy $\boldsymbol{P}$, where $\ell \leq k$. To this end, the algorithm invokes $\mathcal{A}_{\boldsymbol{P}}$ as a subroutine with inputs $\left(\left\langle\kappa, \boldsymbol{f}_{i}\right\rangle, x_{i}\right)$ for each $i \in\{1, \ldots, \ell\}$. If any input is rejected, the algorithm accepts, otherwise it rejects. The running time is obviously bounded by the size of $V\left(\operatorname{body}_{\mathcal{Q}}\right)$ and the represention size of $\kappa$.

In the following proofs, the representation size of nodes is explicitly or implicitly bounded by the length of the input.

Proposition B.2. Problem $\mathrm{PCI}\left(\mathcal{P}_{\text {nrel }}\right)$ is in $\Pi_{2}^{P}$.

Proof. We show that the complement problem $\overline{\mathrm{PCI}\left(\mathcal{P}_{\text {nrel }}\right)}$ is in $\Sigma_{2}^{P}=\mathrm{CO}-\Pi_{2}^{P}$.

By Definition 3.1, a query $\mathcal{Q}$ is not parallel-correct on instance $I$ under distribution policy $\boldsymbol{P}_{n}$ if and only if there is a fact in $\mathcal{Q}(I)$ that cannot be derived on any node. This condition is equivalent to the existence of a valuation $V$ that satisfies $\mathcal{Q}$ on $I$ such that for every valuation $V^{\prime}$ that satisfies $\mathcal{Q}$ on $I$ and derives the same fact, $V\left(\right.$ head $\left._{\mathcal{Q}}\right)=V^{\prime}\left(\right.$ head $\left._{\mathcal{Q}}\right)$, no node in $\operatorname{dom}_{n}$ is assigned all facts from $V^{\prime}\left(\right.$ body $\left._{\mathcal{Q}}\right)$.

This condition, in turn, can be translated straightforwardly into a $\Sigma_{2}^{P}$-algorithm for $\overline{\mathrm{PCI}\left(\mathcal{P}_{\text {nrel }}\right)}$. Valuations that satisfy $\mathcal{Q}$ on $I$ can be represented by a string of length polynomial in $\mathcal{Q}$ and $I$. Further, every node in $\mathbf{d o m}_{n}$ can be represented by a string of length $n$. 
By definition of $\Sigma_{2}^{P}$, it suffices to give an algorithm with polynomial time bound in $\langle\mathcal{Q}, I, n\rangle$ such that there is some valuation $V$ such that for every valuation $V^{\prime}$, every node $\kappa$ and every string $x$ the algorithm accepts input $\left(\langle\mathcal{Q}, I, n\rangle, V,\left\langle V^{\prime}, \kappa, x\right\rangle\right)$ if and only if $(\mathcal{Q}, I, n) \notin \operatorname{PCI}\left(\mathcal{P}_{\text {nrel }}\right)$. In the following, we describe the algorithm and mention the time complexity of its main steps. Correctness of the algorithm results from the characterization above.

First, the algorithm tests whether $V$ and $V^{\prime}$ satisfy $\mathcal{Q}$ on $I$. If $V$ does not satisfy on $I$, the algorithm rejects. If $V^{\prime}$ does not satisfy on $I$, the algorithm accepts. Second, the algorithm tests whether $V$ and $V^{\prime}$ derive the same fact, $V\left(\right.$ head $\left._{\mathcal{Q}}\right)=V^{\prime}\left(\right.$ head $\left._{\mathcal{Q}}\right)$. If they do not, the algorithm accepts. Otherwise it continues. All these tests can obviously be done in time polynomial in $\mathcal{Q}$ and $I$.

We assume $x$ to be a string of the form $x_{1} \circ \cdots \circ x_{k}$, where each $x_{i}$ is a string of length polynomial in $n$ and $k$ is the number of atoms in $\mathcal{Q}$.

Lastly, as described in Remark B.1, the algorithm tests whether there is a fact in $V^{\prime}\left(\right.$ body $\left.y_{\mathcal{Q}}\right)$ that is not assigned to node $\kappa$ and passes the result (if a fact is not assigned to $\kappa$, the algorithm accepts, otherwise it rejects). Since the size of $V^{\prime}\left(\operatorname{bod} y_{\mathcal{Q}}\right)$ is polynomially bounded by $\mathcal{Q}$ and $I$, this step also has a time bound polynomial in $\langle\mathcal{Q}, I, n\rangle$ (recall that $n$ is the representation size of nodes in the network underlying $\boldsymbol{P}_{n}$ ).

Corollary B.3. Problem $\mathrm{PCI}\left(\mathcal{P}_{\text {fin }}\right)$ is in $\Pi_{2}^{P}$.

Proof. Obviously, problem $\mathrm{PCI}\left(\mathcal{P}_{\text {fin }}\right)$ can be polynomially reduced to $\mathrm{PCI}\left(\mathcal{P}_{\text {nrel }}\right)$ by choosing $n$ to be the maximum representation length of any node in $\boldsymbol{P}$. Even though $n$ has to be encoded unary, explicit enumeration of all fact-node assignments in any distribution policy from $\mathcal{P}_{\text {fin }}$ guarantees that the length of $n$ is polynomial in $\boldsymbol{P}$, which is an input parameter for problem $\operatorname{PCI}\left(\mathcal{P}_{\text {fin }}\right)$.

To decide parallel-correctness of a query under a distribution policy for all subinstances of a given instance, we use a minor variation of Lemma 3.4, where parallel-correctness is not required for all possible instances but rather for all subinstances of a given instance.

Lemma B.4. A query $\mathcal{Q}$ is parallel-correct on all subinstances $I^{\prime}$ of an instance $I$ under distribution policy $\boldsymbol{P}$ if and only if for every minimal valuation $V$ that satisfies $\mathcal{Q}$ on $I$ it holds $\bigcap_{\boldsymbol{f} \in V\left(\operatorname{body} y_{\mathcal{Q}}\right)} \boldsymbol{P}(\boldsymbol{f}) \neq \emptyset$.

Proposition B.5. Problem $\mathrm{PC}\left(\mathcal{P}_{\text {nrel }}\right)$ is in $\Pi_{2}^{P}$.

Proof. We show that the complement problem $\overline{\mathrm{PC}\left(\mathcal{P}_{\text {nrel }}\right)}$ is in $\Sigma_{2}^{P}=\mathrm{CO}-\Pi_{2}^{P}$. Now, instance $I$ is an arbitrary instance whose data values can be represented by a string of length $n$.

By Lemma B.4, a query $\mathcal{Q}$ is not parallel-correct on each subinstance $I^{\prime}$ of $I$ under distribution policy $\boldsymbol{P}_{n}$ if there is a minimal valuation $V$ that satisfies $\mathcal{Q}$ on $I$ such that no node in $\mathbf{d o m}_{n}$ is assigned all facts from $V\left(\operatorname{bod}_{\mathcal{Q}}\right)$.

Obviously, valuations can be represented by a string of length polynomial in $\mathcal{Q}$ and $n$. Further, every node in $\mathbf{d o m}_{n}$ can be represented by a string of length $n$.

By definition of $\Sigma_{2}^{P}$, it suffices to give an algorithm with polynomial time bound in $\langle\mathcal{Q}, n\rangle$ such that there is some valuation $V$ such that for every valuation $V^{\prime}$, every node $\kappa$, and every string $x$ the algorithm accepts input $\left(\langle\mathcal{Q}, n\rangle, V,\left\langle V^{\prime}, \kappa, x\right\rangle\right)$ if and only if $(\mathcal{Q}, n) \notin \mathrm{PC}\left(\mathcal{P}_{\text {nrel }}\right)$. In the following we describe the algorithm and mention the time complexity of its main steps. Correctness of the algorithm results from the characterization in Lemma B.4.

First, the algorithm tests whether valuation $V^{\prime}$ contradicts minimality of valuation $V$. For this, it checks whether $V^{\prime}$ derives the same fact, $V^{\prime}\left(\right.$ head $\left._{\mathcal{Q}}\right)=V\left(\right.$ head $\left.d_{\mathcal{Q}}\right)$, and also whether $V^{\prime}\left(\operatorname{bod}_{\mathcal{Q}}\right) \subsetneq V\left(\right.$ body $\left._{\mathcal{Q}}\right)$. If both tests succeed, the algorithm rejects. Otherwise it continues. 
Next, the algorithm tests whether a fact in $V\left(\right.$ body $\left.y_{\mathcal{Q}}\right)=\left\{\boldsymbol{f}_{1}, \ldots, \boldsymbol{f}_{\ell}\right\}$ is not not assigned to node $\kappa$, as described in Remark B.1, and passes the result (if a fact is not assigned to $\kappa$, the algorithm accepts, otherwise it rejects). Since the size of $V\left(\right.$ body $\left.y_{\mathcal{Q}}\right)$ is polynomially bounded by $\mathcal{Q}$ and $n$, this step also has a time bound polynomial in $\langle\mathcal{Q}, n\rangle$.

Corollary B.6. Problem $\mathrm{PC}\left(\mathcal{P}_{\text {fin }}\right)$ is in $\Pi_{2}^{P}$.

Proof. The algorithm for $\mathrm{PC}\left(\mathcal{P}_{\text {nrel }}\right)$ in the proof of Proposition B.5 can be adapted to a decision algorithm for $\operatorname{PC}\left(\mathcal{P}_{\text {fin }}\right)$. Before the other tests, it is checked whether valuations $V, V^{\prime}$ satisfy $\mathcal{Q}$ on facts $(\boldsymbol{P})$. If $V$ does not satisfy $\mathcal{Q}$ on facts $(\boldsymbol{P})$, then the algorithm rejects. If $V^{\prime}$ does not satisfy $\mathcal{Q}$ on facts $(\boldsymbol{P})$, then the algorithm accepts. In any other case, the algorithm continues.

Obviously, both tests can be accomplished in time polynomial in $\langle\mathcal{Q}, \boldsymbol{P}\rangle$.

\section{B.2.2 Lower bounds}

We first prove the lower bound for $\mathrm{PCI}\left(\mathcal{P}_{\text {fin }}\right)$ in Proposition B.7 by a reduction from $\Pi_{2}$-QBF. This reduction can also be adapted to a polynomial reduction to $\mathrm{PC}\left(\mathcal{P}_{\text {fin }}\right)$, Proposition B.8.

Proposition B.7. $\operatorname{PCI}\left(\mathcal{P}_{\text {fin }}\right)$ is $\Pi_{2}^{P}$-hard, even for distribution policies with only two nodes.

Proof. We give a polynomial reduction from the $\Pi_{2}^{P}$-complete problem $\Pi_{2}$-QBF .

Let $\varphi$ be an input for $\Pi_{2}$-QBF, i.e., a formula of the form $\forall \mathbf{x} \exists \mathbf{y} \psi(\mathbf{x}, \mathbf{y})$. We assume $\psi$ to be a propositional formula in 3 -CNF with variables $\mathbf{x}=\left(x_{1}, \ldots, x_{m}\right)$ and $\mathbf{y}=\left(y_{1}, \ldots, y_{n}\right)$. Let $C_{1}, \ldots, C_{k}$ denote their (disjunctive) clauses, where, for each $j, C_{j}=\left(\ell_{j, 1} \vee \ell_{j, 2} \vee \ell_{j, 3}\right)$.

We describe next how the corresponding input instance for $\mathrm{PCI}\left(\mathcal{P}_{\text {fin }}\right)$, consisting of a query $\mathcal{Q}_{\varphi}$, a database instance $I_{\varphi}$, and a distribution policy $\boldsymbol{P}_{\varphi}$ is defined.

The query $\mathcal{Q}_{\varphi}$ is formulated over variables $w_{1}, w_{0}$, and $x_{g}, \bar{x}_{g}, y_{h}, \bar{y}_{h}$, for $g \in\{1, \ldots, m\}$ and $h \in\{1, \ldots, n\}$. Intuitively, these variables are intended to represent the Boolean values true and false and the (negated) values of the variables $x_{g}, y_{h}$ in $\psi$, respectively. We overload the notation $\ell_{j, i}$ as follows: if $\ell_{j, i}$ is a negated literal $\neg x$ in $C_{j}$, then $\ell_{j, i}$ also denotes the variable $\bar{x}$.

Let $\mathbb{B}^{+} \stackrel{\text { def }}{=} \mathbb{B} \backslash\{(0,0,0)\}$ be the set of non-zero Boolean triples and $\mathbb{W}^{+} \stackrel{\text { def }}{=} \mathbb{W} \backslash\left\{\left(w_{0}, w_{0}, w_{0}\right)\right\}$ the set of triples over $\left\{w_{0}, w_{1}\right\}$ that contain at least one $w_{1}$.

We define $\mathcal{Q}_{\varphi}$ to be the query with $h e a d_{\mathcal{Q}_{\varphi}}=H\left(x_{1}, \ldots, x_{m}\right)$ and $\operatorname{body}_{\mathcal{Q}_{\varphi}}=\operatorname{Cons} \cup \operatorname{Struct}(\psi)$, where

$$
\text { Cons } \stackrel{\text { def }}{=}\left\{\operatorname{True}\left(w_{1}\right), \operatorname{Fal} \operatorname{se}\left(w_{0}\right), \operatorname{Neg}\left(w_{1}, w_{0}\right), \operatorname{Neg}\left(w_{0}, w_{1}\right)\right\} \cup\left\{\mathbf{C}_{j}(\mathbf{w}) \mid j \in\{1, \ldots, k\}, \mathbf{w} \in \mathbb{W}^{+}\right\}
$$

is a set of consistency atoms, representing valid combinations of values for Neg-facts and satisfying combinations of values for $\mathrm{C}_{j}$-facts, and

$$
\begin{aligned}
& \operatorname{Struct}(\psi) \stackrel{\text { def }}{=}\left\{\operatorname{Neg}(x, \bar{x}) \mid x \in\left\{x_{1}, \ldots, x_{m}, y_{1}, \ldots, y_{n}\right\}\right\}, \\
& \cup\left\{C_{j}\left(\ell_{j, 1}, \ell_{j, 2}, \ell_{j, 3}\right) \mid \text { for each clause } C_{j}=\left(\ell_{j, 1} \vee \ell_{j, 2} \vee \ell_{j, 3}\right)\right\}
\end{aligned}
$$

is a set of atoms representing the logical structure of $\psi$ : it relates variable $x_{g}$ to $\bar{x}_{g}$ and also variable $y_{h}$ to $\bar{y}_{h}$ for each $g \in\{1, \ldots, m\}$ and $h \in\{1, \ldots, n\}$, respectively. Additionally, it relates all variables that represent literals occurring in the same clause to each other., The database instance $I_{\varphi}$ is defined as

$$
\{\operatorname{True}(1), \text { False }(0), \operatorname{Neg}(1,0), \operatorname{Neg}(0,1)\} \cup\left\{\mathrm{C}_{j}(\mathbf{b}) \mid j \in\{1, \ldots, k\}, \mathbf{b} \in \mathbb{B}\right\} .
$$

We partition it into $I_{\varphi}^{-\stackrel{\text { def }}{=}}\left\{\mathrm{C}_{j}(0,0,0) \mid j \in\{1, \ldots, k\}\right\}$ and $I_{\varphi}^{+} \stackrel{\text { def }}{=} I_{\varphi} \backslash I_{\varphi}^{-}$. 
We define $\boldsymbol{P}_{\varphi}$ to be the finite distribution policy for $I_{\varphi}$ over a network $\mathcal{N}=\left\{\kappa^{+}, \kappa^{-}\right\}$as

$$
\boldsymbol{P}_{\varphi}(\boldsymbol{f})= \begin{cases}\left\{\kappa^{+}\right\} & \text {if } \boldsymbol{f} \in I_{\varphi}^{+} \\ \left\{\kappa^{-}\right\} & \text {if } \boldsymbol{f} \in I_{\varphi}^{-}\end{cases}
$$

Correctness Obviously, query $\mathcal{Q}_{\varphi}$, instance $I_{\varphi}$ and distribution policy $\boldsymbol{P}_{\varphi}$ can be computed in polynomial time from $\varphi$. We will now prove that this mapping is indeed a reduction, that is, that $\left(\mathcal{Q}_{\varphi}, I_{\varphi}, \boldsymbol{P}_{\varphi}\right) \in$ $\operatorname{PCI}\left(\mathcal{P}_{\text {fin }}\right)$ if and only if $\varphi \in \Pi_{2}-\mathrm{QBF}$.

(if) Let $\varphi \in \Pi_{2}$-QBF. We need to show $\left(\mathcal{Q}_{\varphi}, I_{\varphi}, \boldsymbol{P}_{\varphi}\right) \in \mathrm{PCI}\left(\mathcal{P}_{\text {fin }}\right)$. To this end, let $\boldsymbol{f}=H\left(a_{1}, \ldots, a_{m}\right)$ be an arbitrary fact in $\mathcal{Q}_{\varphi}\left(I_{\varphi}\right)$. We show that $\boldsymbol{f} \in \mathcal{Q}_{\varphi}\left(I_{\varphi}^{+}\right)$and thus that $\boldsymbol{f}$ is derived at node $\kappa^{+}$.

Since $\operatorname{adom}(I)=\{0,1\}$, it holds $a_{1}, \ldots, a_{m} \in\{0,1\}$. Therefore, the mapping $\beta_{\mathbf{x}}$ defined by $\beta_{\mathbf{x}}\left(x_{g}\right)=a_{g}$ for each $g \in\{1, \ldots, m\}$ is a well-defined truth assignment. By assumption, there is a truth assignment $\beta_{\mathbf{y}}$ for $y_{1}, \ldots, y_{n}$ such that $\beta \models \psi$, where $\beta \stackrel{\text { def }}{=} \beta_{\mathbf{x}} \cup \beta_{\mathbf{y}}$.

We define valuation $V$ for $\mathcal{Q}_{\varphi}$ by $w_{1} \mapsto 1, w_{0} \mapsto 0$ and $u \mapsto \beta(u), \bar{u} \mapsto \overline{\beta(u)}$ for $u \in\left\{x_{1}, \ldots, x_{m}, y_{1}, \ldots, y_{n}\right\}$, respectively. Since $\beta \models \psi$, in particular, $\beta \models C_{j}$ for every $j \in\{1, \ldots, k\}$. Therefore, for every $j \in\{1, \ldots, k\}$ there is some $\mathbf{b} \in \mathbb{B}^{+}$such that $V\left(\mathrm{C}_{j}\left(\ell_{j, 1}, \ell_{j, 2}, \ell_{j, 3}\right)\right)=\mathrm{C}_{j}(\mathbf{b})$. Hence, all required $\mathrm{C}_{j}$-facts and True-, False-, Neg-facts in $V\left(\operatorname{body}_{\mathcal{Q}_{\varphi}}\right)$ are contained in $I_{\varphi}^{+}$and therefore $H\left(a_{1}, \ldots, a_{m}\right)=V\left(\right.$ head $\left._{\mathcal{Q}_{\varphi}}\right)$ can be derived on node $\kappa^{+}$.

(only-if) Now, let $\varphi \notin \Pi_{2}$-QBF. We show that $\left(\mathcal{Q}_{\varphi}, I_{\varphi}, \boldsymbol{P}_{\varphi}\right) \notin \mathrm{PCI}\left(\mathcal{P}_{\text {fin }}\right)$. By the assumption on $\varphi$ there is a valuation $\beta_{\mathbf{x}}$ for $x_{1}, \ldots, x_{m}$ such that for every valuation $\beta_{\mathbf{y}}$ for $y_{1}, \ldots, y_{n}$ it holds $\left(\beta_{\mathbf{x}} \cup \beta_{\mathbf{y}}\right) \not \forall \psi$.

Let $\boldsymbol{f}=H\left(\beta_{\mathbf{x}}\left(x_{1}\right), \ldots, \beta_{\mathbf{x}}\left(x_{m}\right)\right)$ and $\beta$ be the extension of $\beta_{\mathbf{x}}$ on the variables $y_{1}, \ldots, y_{n}$ defined by $\beta\left(y_{h}\right)=0$ for every $h \in\{1, \ldots, n\}$. This truth assignment induces a valuation $V$ that satisfies $\mathcal{Q}_{\varphi}$ on $I_{\varphi}$ because $\mathrm{C}_{j}(\mathbf{b})$-facts for all tuples $\mathbf{b} \in \mathbb{B}$ are in $I_{\varphi}$. Thus, $\boldsymbol{f} \in \mathcal{Q}_{\varphi}\left(I_{\varphi}\right)$.

Clearly, $\boldsymbol{f}$ can not be derived at node $\kappa^{-}$as, e.g., $I_{\varphi}^{-}$does not contain any Neg-facts. However, $\boldsymbol{f}$ can neither be derived at node $\kappa^{+}$: Towards a contradiction, let us assume that some valuation $V$ yields $f$ at node $\kappa^{+}$. In particular, $V$ needs to map all $C_{j}$-atoms of $\operatorname{Struct}(\psi)$ to facts from $I_{\varphi}^{+}$. But then $V$ induces a truth assignment $\beta_{\mathbf{y}}$ on variables $y_{1}, \ldots, y_{n}$ with $\left(\beta_{\mathbf{x}} \cup \beta_{\mathbf{y}}\right) \models \psi$, contradicting the choice of $\beta_{\mathbf{x}}$.

Proposition B.8. Problem $\mathrm{PC}\left(\mathcal{P}_{\text {fin }}\right)$ is $\Pi_{2}^{P}$-hard, even for distribution policies with only two nodes.

Proof. Again, we give a polynomial reduction from the $\Pi_{2}^{P}$-complete problem $\Pi_{2}$-QBF. Let $\varphi$ be an input formula for $\Pi_{2}$-QBF. The (intended) reduction maps $\varphi$ to the query $\mathcal{Q}_{\varphi}$ and the distribution policy $\boldsymbol{P}_{\varphi}$, as defined in the proof of Proposition B.7.

This mapping is clearly computable in polynomial time and we show next that it is a reduction, that is, $\left(\mathcal{Q}_{\varphi}, \boldsymbol{P}_{\varphi}\right) \in \mathrm{PC}\left(\mathcal{P}_{\text {fin }}\right)$ if and only if $\varphi \in \Pi_{2}$-QBF.

(if) Let $\varphi \in \Pi_{2}$-QBF. We have to show that $\mathcal{Q}_{\varphi}$ is parallel-correct on all input instances under $\boldsymbol{P}_{\varphi}$.

Let $I$ be such an instance. As True(1) and False(0) are the only facts under $\boldsymbol{P}_{\varphi}$ for relation symbols

True and False, respectively, every satisfying valuation for $\mathcal{Q}_{\varphi}$ needs to map $w_{0} \mapsto 0$ and $w_{1} \mapsto 1$.

If $I_{\varphi}^{+} \nsubseteq I$, then $\mathcal{Q}_{\varphi}(I)=\emptyset$ because every satisfying valuation for $\mathcal{Q}_{\varphi}$ requires all facts from $I_{\varphi}^{+}$to satisfy the set Cons. Thus, in this case, $\mathcal{Q}_{\varphi}$ is parallel-correct on $I$ under distribution policy $\boldsymbol{P}_{\varphi}$.

If $I_{\varphi}^{+} \subseteq I$, we have $I_{\varphi}^{+} \subseteq I \subseteq I_{\varphi}$. In the proof of Proposition B.7 it was shown that $\mathcal{Q}_{\varphi}\left(I_{\varphi}^{+}\right)=\mathcal{Q}_{\varphi}\left(I_{\varphi}\right)$. Monotonicity yields $\mathcal{Q}_{\varphi}\left(I_{\varphi}^{+}\right)=\mathcal{Q}_{\varphi}(I)$ and therefore all facts in $\mathcal{Q}_{\varphi}(I)$ are produced at node $\kappa^{+}$. 
(only-if) Let $\varphi \notin \Pi_{2}$-QBF. It was shown in the proof of Proposition B.7 that in this case $\mathcal{Q}_{\varphi}$ is not parallel-correct on instance $I_{\varphi}$ under $\boldsymbol{P}_{\varphi}$ and, in particular, it is not parallel-correct under $\boldsymbol{P}_{\varphi}$.

\section{Proofs for Section 4: Transferability}

\section{C.1 Proof of Lemma 4.2 .}

The two implications of Lemma 4.2 are shown in Propositions C.1 and C.2 below.

Proposition C.1. Let $\mathcal{Q}$ and $\mathcal{Q}^{\prime}$ be CQs. If condition (C2) holds, then, parallel-correctness transfers from $\mathcal{Q}$ to $\mathcal{Q}^{\prime}$.

Proof. Let $\boldsymbol{P}$ be a distribution policy under which $\mathcal{Q}$ is parallel-correct and let $I$ be an instance. Then we show that $\mathcal{Q}^{\prime}$ is parallel-correct as well on $I$ under $\boldsymbol{P}$. By monotonicity of CQs, $\bigcup_{x \in \mathcal{N}} \mathcal{Q}^{\prime}\left(\operatorname{dist}_{\boldsymbol{P}}(I)(x)\right) \subseteq$ $\mathcal{Q}^{\prime}(I)$. Thus it suffices to show that for every fact $f \in \mathcal{Q}^{\prime}(I)$, there is some valuation for $\mathcal{Q}^{\prime}$ that allows to derive $\boldsymbol{f}$ on one of the chunks of $I$ under $\boldsymbol{P}$. For $\boldsymbol{f} \in \mathcal{Q}^{\prime}(I)$, there is a minimal valuation $V^{\prime}$ for $\mathcal{Q}^{\prime}$ which satisfies on $I$ for $\mathcal{Q}^{\prime}$ and derives $\boldsymbol{f}$. That is, $V^{\prime}\left(\operatorname{body}_{\mathcal{Q}^{\prime}}\right) \subseteq I$ and $V^{\prime}\left(\right.$ head $\left._{\mathcal{Q}^{\prime}}\right)=\boldsymbol{f}$. Next, we show that the facts required by $V^{\prime}$ for $\mathcal{Q}^{\prime}$ meet at some node under $\boldsymbol{P}$, which implies that the chunks of $I$ under $\boldsymbol{P}$ indeed allow deriving $\boldsymbol{f}$.

For this, we rely on the assumption that there is a minimal valuation $V$ for $\mathcal{Q}$, where $V^{\prime}\left(b o d y_{\mathcal{Q}^{\prime}}\right) \subseteq$ $V\left(\operatorname{bod} y_{\mathcal{Q}}\right)$. Let $J=V\left(\right.$ bod $\left.y_{\mathcal{Q}}\right)$. Then, by parallel-correctness of $\mathcal{Q}$ under $\boldsymbol{P}$, there is a valuation $W$ and node $\kappa \in \mathcal{N}$, such that $W\left(\right.$ body $\left._{\mathcal{Q}}\right) \subseteq \operatorname{dist}_{\boldsymbol{P}}(J)(\kappa)$ and $W\left(\right.$ head $\left._{\mathcal{Q}}\right)=V\left(\right.$ head $\left._{\mathcal{Q}}\right)$. Because $V$ is minimal and $\operatorname{dist}_{\boldsymbol{P}}(J)(\kappa) \subseteq V\left(\right.$ body $\left._{\mathcal{Q}}\right)$, it must be that $V\left(\operatorname{bod}_{\mathcal{Q}}\right)=W\left(\right.$ body $\left.y_{\mathcal{Q}}\right)$. So, $\boldsymbol{P}$ maps all the facts in $J$ onto node $\kappa$, implying that all the facts in $V^{\prime}\left(\operatorname{bod}_{\mathcal{Q}^{\prime}}\right)$ are mapped onto node $\kappa$ under $\boldsymbol{P}$ (because $V^{\prime}\left(\right.$ bod $\left.y_{\mathcal{Q}^{\prime}}\right) \subseteq$ $\left.V\left(\operatorname{bod} y_{\mathcal{Q}}\right)=J\right)$.

Hence, $\mathcal{Q}^{\prime}$ is indeed parallel-correct under the distribution policies for which $\mathcal{Q}$ is parallel-correct.

Proposition C.2. Let $\mathcal{Q}$ and $\mathcal{Q}^{\prime}$ be CQs. If parallel-correctness transfers from $\mathcal{Q}$ to $\mathcal{Q}^{\prime}$, then, condition (C2) holds.

Proof. The proof is by contraposition. So, we assume that there is a minimal valuation $V^{\prime}$ for $\mathcal{Q}^{\prime}$ for which there is no valuation $V$ for $\mathcal{Q}$, where $V^{\prime}\left(\operatorname{bod}_{\mathcal{Q}^{\prime}}\right) \subseteq V\left(\operatorname{bod} y_{\mathcal{Q}}\right)$. Let $m=\left|V^{\prime}\left(\operatorname{bod} y_{\mathcal{Q}^{\prime}}\right)\right|$.

We distinguish two cases, depending on whether $V^{\prime}$ requires only one fact or at least two facts. For both cases we construct a network $\mathcal{N}$ and distribution policy $\boldsymbol{P}$ over $\mathcal{N}$ for which $\mathcal{Q}$ is parallel-correct but $\mathcal{Q}^{\prime}$ is not, implying that parallel-correctness does not transfer from $\mathcal{Q}$ to $\mathcal{Q}^{\prime}$.

1. Case $(m=1)$ : Let $V^{\prime}\left(\right.$ body $\left._{\mathcal{Q}^{\prime}}\right)=\{\boldsymbol{f}\}$. Let $\mathcal{N}$ be a single-node network, i.e., $\mathcal{N} \stackrel{\text { def }}{=}\{\kappa\}$. For $\boldsymbol{P}$ we consider the distribution policy thats skips $\boldsymbol{f}$, that is, maps $\boldsymbol{P}(\boldsymbol{f})$ to the empty set, and maps every other fact in $\operatorname{facts}(\mathcal{D})$ onto node $\kappa$. By assumption on $V^{\prime}$, none of the minimal valuations for $\mathcal{Q}$ requires $\boldsymbol{f}$. So it immediately follows by Lemma 3.4 that $\mathcal{Q}$ is parallel-correct under $\boldsymbol{P}$. However, because $V^{\prime}$ is minimal for $\mathcal{Q}^{\prime}, \mathcal{Q}^{\prime}$ needs $\boldsymbol{f}$ to derive $V\left(\right.$ head $\left._{\mathcal{Q}^{\prime}}\right)$ when only $\boldsymbol{f}$ is given as input instance. Thus $\mathcal{Q}^{\prime}$ is not parallel-correct under $\boldsymbol{P}$ which leads to the desired contradiction.

2. Case $(m \geq 2)$ : Let $I \stackrel{\text { def }}{=} V^{\prime}\left(b_{o d y_{Q^{\prime}}}\right)=\left\{\boldsymbol{f}_{1}, \ldots, \boldsymbol{f}_{m}\right\}, \mathcal{N} \stackrel{\text { def }}{=}\left\{\kappa_{1}, \ldots, \kappa_{m}\right\}$, and let $\boldsymbol{P}$ be the mapping defined as follows:

- $\boldsymbol{P}(\boldsymbol{g})=\mathcal{N}$, for every $\boldsymbol{g} \in \operatorname{facts}(\mathcal{D}) \backslash I$; and

- $\boldsymbol{P}\left(\boldsymbol{f}_{i}\right)=\mathcal{N} \backslash\left\{\kappa_{i}\right\}$, for every $i$. 
Intuitively, on every instance $J$, either the facts in $J$ meet on some node under $\boldsymbol{P}$, or $I \subseteq J$. By assumption, none of the minimal valuations for $\mathcal{Q}$ requires all the facts in $I$, implying that $\mathcal{Q}$ is parallelcorrect under $\boldsymbol{P}$. Nevertheless, on instance $I$ under $\boldsymbol{P}$, none of the nodes receives all the facts in $I$, and there is no valuation that can derive $V^{\prime}\left(\right.$ head $\left._{\mathcal{Q}^{\prime}}\right)$ for a strict subset of the facts in $I$ (by minimality of $\left.V^{\prime}\right)$. So, $\mathcal{Q}^{\prime}$ is not parallel-correct under $\boldsymbol{P}$ which leads to the desired contradiction.

Remark C.3. The reader may wonder what the effect is on Lemma 4.2 when distribution policies are not allowed to skip facts. In fact, not much changes, except that we now need to deal explicitly with the trivial case where a valuation for $\mathcal{Q}^{\prime}$ requires only one fact. More specifically, in this case, $\mathcal{Q}^{\prime}$ is parallel-correct under all the distribution policies where $\mathcal{Q}$ is parallel-correct for if and only if

(C2') for every minimal valuation $V^{\prime}$ for $\mathcal{Q}^{\prime}$, either $V^{\prime}$ requires only one fact, or there is a minimal valuation $V$ for $\mathcal{Q}$ such that $V^{\prime}\left(b o d y_{\mathcal{Q}^{\prime}}\right) \subseteq V\left(\operatorname{bod}_{\mathcal{Q}}\right)$.

The proof is similar to the proof for Proposition C.1 and Proposition C.2, except that when condition (C2') fails, a counterexample must require at least two facts, and thus case 1 in the proof of Proposition C.2 drops. Notice that the distribution policy given in case 2 does not skip any facts, and is thus well-defined in the adapted setting as well.

\section{C.2 Proof of Theorem 4.3}

In principle, Lemma 4.2, on which the following proofs are based, talks about an infinite number of valuations over the infinite domain dom. However, since our queries are generic, the only observable property of the constants used by some valuation is equality/inequality. It therefore suffices to check valuations over an arbitrary finite domain with at least as much constants as valuations for both queries can use. This is stated more explicitly in the following claim.

Claim C.4. Let $\mathcal{Q}$ and $\mathcal{Q}^{\prime}$ be CQs with variables $x_{1}, \ldots, x_{m}$ and $y_{1}, \ldots, y_{n}$, respectively. Moreover, for $k=m+n$ let $d_{0} m_{k}=\{1, \ldots, k\}$ be a subset of the (countably) infinite set dom.

The following two conditions are equivalent.

1. For every minimal valuation $V^{\prime}$ for $\mathcal{Q}^{\prime}$ over $\operatorname{dom}$ there is a minimal valuation $V$ for $\mathcal{Q}$ over $\operatorname{dom}$ such that $V^{\prime}\left(\operatorname{bod}_{\mathcal{Q}^{\prime}}\right) \subseteq V\left(\operatorname{bod}_{\mathcal{Q}}\right)$.

2. For every minimal valuation $V^{\prime}$ for $\mathcal{Q}^{\prime}$ over $d_{0} m_{k}$ there is a minimal valuation $V$ for $\mathcal{Q}$ over $d o m_{k}$ such that $V^{\prime}\left(\right.$ bod $\left.y_{\mathcal{Q}^{\prime}}\right) \subseteq V\left(\right.$ body $\left.y_{\mathcal{Q}}\right)$.

Completeness of parallel-correctness transfer for general distribution policies follows from the upper bound stated in Proposition C.5 and the lower bound stated in Proposition C.6.

Proposition C.5. PC-TRANS $\in \Pi_{3}^{P}$.

Proof. By Lemma 4.2, deciding parallel-correctness transfer is equivalent to verifying that for each minimal valuation $V^{\prime}$ for $\mathcal{Q}^{\prime}$ there is a minimal valuation $V$ for $\mathcal{Q}$ such that $V^{\prime}\left(\right.$ body $\left.\mathcal{Q}_{\mathcal{Q}^{\prime}}\right) \subseteq V\left(\right.$ body $\left._{\mathcal{Q}}\right)$. This, in turn, is equivalent to checking for each valuation $V^{\prime}$ for $\mathcal{Q}^{\prime}$ that it is not minimal, which can be witnessed by another valuation $W^{\prime}$ that derives the same fact and requires strictly less facts, or that there is a minimal 
valuation $V$ for $\mathcal{Q}$ such that $V^{\prime}\left(\operatorname{bod}_{\mathcal{Q}_{\varphi}^{\prime}}\right) \subseteq V\left(\operatorname{bod}_{\mathcal{Q}_{\varphi}}\right)$. Non-minimality of valuation $V$ can be witnessed by a valuation $W$. Due to Claim C.4, all valuations can be restricted to $d_{0} m_{k}=\{1, \ldots, k\}$, where $k=m+n$ and $\mathcal{Q}, \mathcal{Q}^{\prime}$ are queries over variables $x_{1}, \ldots, x_{m}$ and $y_{1}, \ldots, y_{n}$, respectively.

To prove membership in class $\Pi_{3}^{P}$, it suffices to show that there is an algorithm with a time bound polynomial in $|\mathcal{Q}|+\left|\mathcal{Q}^{\prime}\right|$ such that for every pair $\left(\mathcal{Q}, \mathcal{Q}^{\prime}\right)$ of queries it holds $\left(\mathcal{Q}, \mathcal{Q}^{\prime}\right) \in$ PC-TRANs if and only if for every $\mathcal{Q}^{\prime}$-valuation $V^{\prime}$ there is a $\mathcal{Q}$-valuation $V$ and a $\mathcal{Q}^{\prime}$-valuation $W^{\prime}$ such that for every $\mathcal{Q}$-valuation $W$, the algorithm accepts $\left(\left\langle\mathcal{Q}, \mathcal{Q}^{\prime}\right\rangle, V^{\prime},\left\langle V, W^{\prime}\right\rangle, W\right)$.

For input $\left(\left\langle\mathcal{Q}, \mathcal{Q}^{\prime}\right\rangle, V^{\prime},\left\langle V, W^{\prime}\right\rangle, W\right)$ the algorithm proceeds as follows. First, it is checked whether $W^{\prime}$ contradicts the assumed minimality of $V^{\prime}$, that is, whether $W^{\prime}\left(\right.$ hea $\left._{\mathcal{Q}^{\prime}}\right)=V^{\prime}\left(\right.$ head $\left._{\mathcal{Q}^{\prime}}\right)$ as well as $W^{\prime}\left(\right.$ bod $\left._{\mathcal{Q}^{\prime}}\right) \subsetneq V^{\prime}\left(\right.$ bod $\left.y_{\mathcal{Q}^{\prime}}\right)$. If this test succeeds, the algorithm accepts because there is no requirement on a non-minimal $\mathcal{Q}^{\prime}$-valuation. Second, it is checked in an analogous fashion whether $W$ contradicts the assumed minimality of $V$. If this test succeeds, the algorithm rejects.

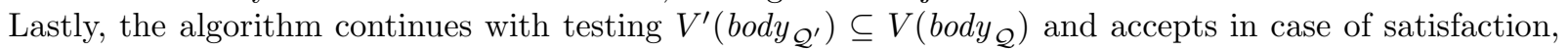
and rejects otherwise. All containment tests can be done in polynomial time.

Proposition C.6. PC-TRANS is $\Pi_{3}^{P}$-hard.

Proof. We give a polynomial reduction from $\Pi_{3}-\mathrm{QBF}$ to PC-TRANS. The proof is based on the characterization of parallel-correctness transfer by condition $(\mathrm{C} 2)$ as stated in Lemma 4.2 .

Reduction function Let thus $\varphi=\forall \mathbf{x} \exists \mathbf{y} \forall \mathbf{z} \psi(\mathbf{x}, \mathbf{y}, \mathbf{z})$ be a formula with a quantifier-free propositional formula $\psi$ in 3 -DNF over variables $\mathbf{x}=\left(x_{1}, \ldots, x_{m}\right), \mathbf{y}=\left(y_{1}, \ldots, y_{n}\right)$, and $\mathbf{z}=\left(z_{1}, \ldots, z_{p}\right)$.

Let $k$ be the number of clauses of $\psi$ and, for each $j \in\{1, \ldots, k\}$, let $C_{j}=\left(\ell_{j, 1} \wedge \ell_{j, 2} \wedge \ell_{j, 3}\right)$ denote the $j$-th (conjunctive) clause of $\psi$.

The reduction function maps $\varphi$ to a pair $\left(\mathcal{Q}_{\varphi}, \mathcal{Q}_{\varphi}^{\prime}\right)$ of CQs that will be described next. It will be obvious that this mapping can be computed in polynomial time. Similarly as in the proof of Proposition B.7, $\mathcal{Q}_{\varphi}$ uses the variables $w_{1}, w_{0}$, which are intended to represent truth and falseness, respectively, the variables of $\psi$ and variables $\bar{u}$, for each variable $u$ of $\psi$, representing the literal $\neg u 8$ Furthermore, it uses the following variables

- $s_{j}$, for every $j \in\{1, \ldots, k\}$, intended to represent the truth value of $C_{j}$, and

- $r_{j}$, for every $j \in\{1, \ldots, k\}$, intended to represent the truth value of $C_{1} \vee \cdots \vee C_{j}$.

We first describe the general construction, give an example explaining its intuition afterwards and finally prove correctness of the reduction.

The queries $\mathcal{Q}_{\varphi}^{\prime}$ and $\mathcal{Q}_{\varphi}$ are defined as follows:

$$
\begin{aligned}
& \text { head }_{\mathcal{Q}_{\varphi}^{\prime}} \stackrel{\text { def }}{=} H\left(x_{1}, \ldots, x_{m}, w_{1}, w_{0}\right) \\
& \text { body }_{\mathcal{Q}_{\varphi}^{\prime}} \stackrel{\text { def }}{=}\left\{\operatorname{YVal}_{1}\left(w_{1}\right), \mathrm{YVal}_{1}\left(w_{0}\right), \ldots, \mathrm{YVal}_{n}\left(w_{1}\right), \operatorname{YVal}_{n}\left(w_{0}\right)\right\} \cup\left\{\operatorname{Res}\left(w_{1}\right)\right\} \\
& \cup \text { Fix; } \\
& \operatorname{head}_{\mathcal{Q}_{\varphi}} \stackrel{\text { def }}{=} H\left(x_{1}, \ldots, x_{m}, y_{1}, \ldots, y_{n}, w_{1}, w_{0}\right) \\
& b o d y_{\mathcal{Q}_{\varphi}} \stackrel{\text { def }}{=}\left\{\operatorname{YVal}_{1}\left(y_{1}\right), \mathrm{YVal}_{1}\left(\bar{y}_{1}\right), \ldots, \mathrm{YVal}_{n}\left(y_{n}\right), \mathrm{YVal}_{n}\left(\bar{y}_{n}\right)\right\} \cup\left\{\operatorname{Res}\left(w_{0}\right), \operatorname{Res}\left(r_{k}\right)\right\} \\
& \cup \quad \text { Fix } \cup \text { Gates } \cup \text { Circuit, }
\end{aligned}
$$

\footnotetext{
${ }^{8}$ Again, if $\ell$ is a negated literal $\neg u$, we write $\ell$ also for $\bar{u}$.
} 
where

$$
\text { Fix } \stackrel{\text { def }}{=}\left\{\operatorname{XVal}_{1}\left(x_{1}\right), \ldots, \mathrm{XVal}_{m}\left(x_{m}\right), \operatorname{True}\left(w_{1}\right), \operatorname{False}\left(w_{0}\right)\right\}
$$

is intended to "fix" truth values for $x_{1}, \ldots, x_{m}, w_{1}, w_{0}$, the set

$$
\text { Gates } \begin{aligned}
\stackrel{\text { def }}{=} & \left\{\operatorname{Neg}\left(w_{0}, w_{1}\right), \operatorname{Neg}\left(w_{1}, w_{0}\right)\right\} \\
\cup \quad \operatorname{And}\left(w_{1}, w_{1}, w_{1}, w_{1}\right), \operatorname{And}\left(w_{0}, w_{1}, w_{1}, w_{0}\right), \operatorname{And}\left(w_{1}, w_{0}, w_{1}, w_{0}\right), \operatorname{And}\left(w_{0}, w_{0}, w_{1}, w_{0}\right), & \left.\quad \operatorname{And}\left(w_{1}, w_{1}, w_{0}, w_{0}\right), \operatorname{And}\left(w_{0}, w_{1}, w_{0}, w_{0}\right), \operatorname{And}\left(w_{1}, w_{0}, w_{0}, w_{0}\right), \operatorname{And}\left(w_{0}, w_{0}, w_{0}, w_{0}\right)\right\} \\
\cup \quad & \left.\operatorname{Or}\left(w_{1}, w_{1}, w_{1}\right), \operatorname{Or}\left(w_{0}, w_{1}, w_{1}\right), \operatorname{Or}\left(w_{1}, w_{0}, w_{1}\right), \operatorname{Or}\left(w_{0}, w_{0}, w_{0}\right)\right\}
\end{aligned}
$$

contains all atoms that are consistent with respect to the intended meaning of negation, And- and Or-gates 9 on $w_{1}, w_{0}$, and

$$
\begin{array}{rll}
\text { Circuit } & \stackrel{\text { def }}{=} & \{\operatorname{Neg}(u, \bar{u}) \mid \text { for each variable } u \text { in } \psi\} \\
\cup & \left\{\operatorname{And}\left(\ell_{j, 1}, \ell_{j, 2}, \ell_{j, 3}, s_{j}\right) \mid \text { for each clause } C_{j}=\left(\ell_{j, 1} \wedge \ell_{j, 2} \wedge \ell_{j, 3}\right)\right\} \\
\cup & \left\{\operatorname{Or}\left(s_{1}, s_{1}, r_{1}\right), \operatorname{Or}\left(r_{1}, s_{2}, r_{2}\right), \ldots, \operatorname{Or}\left(r_{k-1}, s_{k}, r_{k}\right)\right\}
\end{array}
$$

is intended to represent a Boolean circuit (with output bit $r_{k}$ ) that evaluates $\psi$.

Example C.7. For formula $\varphi=\forall x_{1} \exists y_{1} \exists y_{2} \forall z_{1}\left(\left(x_{1} \wedge y_{1} \wedge z_{1}\right) \vee\left(\neg x_{1} \wedge y_{2} \wedge z_{1}\right)\right)$ we obtain the queries

$$
\begin{aligned}
\mathcal{Q}_{\varphi}^{\prime}: H\left(x_{1}, w_{1}, w_{0}\right) \leftarrow & \operatorname{YVal}_{1}\left(w_{1}\right), \operatorname{YVal}_{1}\left(w_{0}\right), \operatorname{YVal}_{2}\left(w_{1}\right), \operatorname{YVal}_{2}\left(w_{0}\right), \operatorname{Res}\left(w_{1}\right), \\
& \operatorname{XVal}_{1}\left(x_{1}\right), \operatorname{True}\left(w_{1}\right), \operatorname{False}\left(w_{0}\right) .
\end{aligned}
$$

and

$$
\begin{aligned}
\mathcal{Q}_{\varphi}: H\left(x_{1}, x_{2}, y_{1}, w_{1}, w_{0}\right) \leftarrow \quad & \operatorname{YVal}_{1}\left(y_{1}\right), \operatorname{YVal}_{1}\left(\bar{y}_{1}\right), \operatorname{YVal}_{2}\left(y_{2}\right), \operatorname{YVal}_{2}\left(\bar{y}_{2}\right), \operatorname{Res}\left(w_{0}\right), \operatorname{Res}\left(r_{2}\right), \\
& \operatorname{XVal}_{1}\left(x_{1}\right), \operatorname{True}\left(w_{1}\right), \operatorname{False}\left(w_{0}\right) \\
& \ldots \text { all atoms from Gates } \ldots, \\
& \operatorname{Neg}\left(x_{1}, \bar{x}_{1}\right), \operatorname{Neg}\left(y_{1}, \bar{y}_{1}\right), \operatorname{Neg}\left(y_{2}, \bar{y}_{2}\right), \operatorname{Neg}\left(z_{1}, \bar{z}_{1}\right), \\
& \operatorname{And}\left(x_{1}, y_{1}, z_{1}, s_{1}\right), \operatorname{And}\left(\bar{x}_{1}, y_{2}, z_{1}, s_{2}\right), \operatorname{Or}\left(s_{1}, s_{1}, r_{1}\right), \operatorname{Or}\left(r_{1}, s_{2}, r_{2}\right)
\end{aligned}
$$

Note that $\varphi \notin \Pi_{3}-\mathrm{QBF}$ because no truth assignment with $z_{1} \mapsto 0$ is satisfying for $\psi$. In particular, for the truth assignment $\beta_{\mathbf{x}}: x_{1} \mapsto 1$ there is no truth assignment $\beta_{\mathbf{y}}$ such that for every $\beta_{\mathbf{z}}$ it holds $\left(\beta_{\mathbf{x}} \cup \beta_{\mathbf{y}} \cup \beta_{\mathbf{z}}\right) \models \psi$. We illustrate why $\left(\mathcal{Q}_{\varphi}, \mathcal{Q}_{\varphi}^{\prime}\right) \notin$ PC-TRANS.

Let $V^{\prime}$ be the valuation for $\mathcal{Q}_{\varphi}^{\prime}$ defined by $V^{\prime}\left(x_{1}\right) \stackrel{\text { def }}{=} \beta_{\mathbf{x}}\left(x_{1}\right)=1, V^{\prime}\left(w_{1}\right) \stackrel{\text { def }}{=} 1$ and $V^{\prime}\left(w_{0}\right) \stackrel{\text { def }}{=} 0$. This valuation is minimal for $\mathcal{Q}_{\varphi}^{\prime}$ because $\mathcal{Q}_{\varphi}^{\prime}$ is full. We get $V^{\prime}\left(\right.$ body $\left.\mathcal{Q}_{\varphi}^{\prime}\right)=$ $\left\{\operatorname{YVal}_{1}(1), \mathrm{YVal}_{1}(0), \operatorname{Res}(1), \mathrm{XVal}_{1}(1), \operatorname{True}(1), \operatorname{False}(0)\right\}$.

We now argue why there is no minimal valuation $V$ for $\mathcal{Q}_{\varphi}$ such that $V^{\prime}\left(\operatorname{bod} y_{\mathcal{Q}_{\varphi}^{\prime}}\right) \subseteq V\left(\operatorname{bod} y_{\mathcal{Q}_{\varphi}}\right)$. If a valuation $V$ fulfills $V^{\prime}\left(\operatorname{bod}_{\mathcal{Q}_{\varphi}^{\prime}}\right) \subseteq V\left(\operatorname{bod}_{\mathcal{Q}_{\varphi}}\right)$ it must map $w_{0} \mapsto 0, w_{1} \mapsto 1, x_{1} \mapsto 1, r_{2} \mapsto 1$. Furthermore, it must map each of $\left(y_{1}, \bar{y}_{1}\right)$ and $\left(y_{2}, \bar{y}_{2}\right)$ to some pair in $\{(0,1),(1,0)\}$. Thus, $V$ induces a truth assignment $\beta_{\mathbf{y}}$ via $\beta_{\mathbf{y}}\left(y_{1}\right) \stackrel{\text { def }}{=} V\left(y_{1}\right)$ and $\beta_{\mathbf{y}}\left(y_{2}\right) \stackrel{\text { def }}{=} V\left(y_{2}\right)$. Let $V^{*}$ be the valuation that coincides with $V$ on all variables $w_{0}, w_{i}, x_{1}, \bar{x}_{1}, y_{1}, \bar{y}_{1}, y_{2}, \bar{y}_{2}$ and maps $z_{1} \mapsto 0$ and maps all other variables to the "correct" values with respect to the semantics of the logicals gates in $\mathcal{Q}_{\varphi}$. In particular, since $\left(\beta_{\mathbf{x}} \cup \beta_{\mathbf{y}} \cup \beta_{\mathbf{z}}\right) \forall \psi\left(\right.$ where $\left.\beta_{\mathbf{z}}\left(z_{1}\right) \stackrel{\text { def }}{=} 0\right)$, we get $V^{*}\left(r_{2}\right)=0$. It is now easy to check that $V^{*}<_{\mathcal{Q}} V$, and therefore that $V$ is not minimal.

\footnotetext{
${ }^{9}$ The last position in a gate-atom represents the output bit of the gate, the others the input bits.
} 
To complete the proof, we need to show the mapping $\varphi \mapsto\left(\mathcal{Q}_{\varphi}, \mathcal{Q}_{\varphi}^{\prime}\right)$ is indeed a reduction.

We start by some observations. We call a valuation $V$ of $\mathcal{Q}_{\varphi} 0$-1-valued, if its range is $\{0,1\}$ and it maps $\left(w_{0}, w_{1}\right)$ to $(0,1)$ and every pair $(u, \bar{u})$ of variables from $\psi$ to $(0,1)$ or $(1,0)$. A 0 -1-valued valuation is called consistent, if the values $V\left(s_{j}\right)$ and $V\left(r_{j}\right)$, for $j \in\{1, \ldots, k\}$ are consistent with the values $V(u)$ for variables of $\psi$, in the obvious sense. That is, $V\left(s_{j}\right)=1$ if and only clause $C_{j}$ evaluates to true for the truth assignment $\beta_{V}$ obtained from $V$ and $V\left(r_{j}\right)=1$ iff $C_{1} \vee \cdots \vee C_{j}$ evaluates to true.

It is easy to see that a 0-1-valued valuation $V$ is consistent, if and only if $V$ (Circuit) $\subseteq V$ (Gates), because inconsistency requires facts in $V$ (Circuit) that are not in $V$ (Gates) and likewise the existence of such facts implies inconsistency.

Claim C.8. For every 0-1-valued valuation $V$ of $\mathcal{Q}_{\varphi}$ the following conditions are equivalent.

(i) $V$ is minimal;

(ii) $V$ is consistent.

To show that (i) implies (ii), let $V$ be a 0 -1-valued minimal valuation. Let $V^{*}$ be the uniquely determined 0 -1-valued consistent valuation that agrees with $V$ on all variables of $\psi$. As $V^{*}$ is consistent, $V^{*}($ Circuit $) \subseteq$ $V^{*}($ Gates $)$. Since $V$ and $V^{*}$ agree on all variables of $\psi$ and $V$ is minimal, we get $V($ Circuit $) \subseteq V^{*}($ Gates $)=$ $V$ (Gates) and thus $V$ is consistent.

To show that (ii) implies (i), let $V$ be a 0 -1-valued consistent valuation. On the other hand, consistency implies $V$ (Circuit) $\subseteq V$ (Gates). Towards a contradiction, let us assume, there is a valuation $V^{*}$ with $V^{*}<_{\mathcal{Q}} V$. As $V$ and $V^{*}$ agree on $w_{0}, w_{1}$ and all variables $x_{i}, y_{i}$, the only possible fact that could account for the strictness in $V^{*}\left(\operatorname{body}_{\mathcal{Q}_{\varphi}}\right) \subsetneq V\left(\operatorname{bod} y_{\mathcal{Q}_{\varphi}}\right)$ is $\operatorname{Res}(1)$, in case $V\left(r_{k}\right)=1$ and $V^{*}\left(r_{k}\right)=0$. However, $V^{*}($ Circuit $) \subseteq V$ (Circuit) $\subseteq V$ (Gates $)=V^{*}$ (Gates $)$, where the middle inclusion holds because $V$ is consistent. Thus, $V^{*}$ is also consistent, and therefore $V^{*}\left(r_{k}\right)=V\left(r_{k}\right)$, the desired contradiction.

Now, we are prepared to prove that $\varphi$ is in $\Pi_{3}$-QBF if and only if parallel-correctness transfers from $\mathcal{Q}_{\varphi}$ to $\mathcal{Q}_{\varphi}^{\prime}$.

(only-if) Let $\varphi=\forall \mathbf{x} \exists \mathbf{y} \forall \mathbf{z} \psi(\mathbf{x}, \mathbf{y}, \mathbf{z})$ be a formula with a quantifier-free propositional formula $\psi$ in 3DNF such that $\varphi \notin \Pi_{3}-\mathrm{QBF}$. We show that there is a minimal valuation $V^{\prime}$ for $\mathcal{Q}_{\varphi}^{\prime}$ such that each valuation $V$ for $\mathcal{Q}_{\varphi}$ which satisfies $V^{\prime}\left(\operatorname{bod}_{\mathcal{Q}_{\varphi}^{\prime}}\right) \subseteq V\left(\right.$ body $\left._{\mathcal{Q}_{\varphi}}\right)$ is not minimal. From that we can conclude by Lemma 4.2 that parallel-correctness does not transfer from $\mathcal{Q}_{\varphi}$ to $\mathcal{Q}_{\varphi}^{\prime}$.

Let $\beta_{\mathbf{x}}$ be a truth assignment for $x_{1}, \ldots, x_{m}$ in $\psi$ such that for all truth assignments $\beta_{\mathbf{y}}$ for $y_{1}, \ldots, y_{n}$ in $\psi$ there is a truth assignment $\beta_{\mathbf{z}}$ for $z_{1}, \ldots, z_{p}$ such that $\left(\beta_{\mathbf{x}} \cup \beta_{\mathbf{y}} \cup \beta_{\mathbf{z}}\right) \not \forall \psi$.

Let $V^{\prime}$ be the valuation defined by $V^{\prime}\left(x_{1}, \ldots, x_{m}, w_{1}, w_{0}\right) \stackrel{\text { def }}{=}\left(\beta_{\mathbf{x}}\left(x_{1}\right), \ldots, \beta_{\mathbf{x}}\left(x_{m}\right), 1,0\right)$, which is minimal for $\mathcal{Q}_{\varphi}^{\prime}$ because $\mathcal{Q}_{\varphi}^{\prime}$ is full.

Let $V$ be any valuation for $\mathcal{Q}_{\varphi}$ such that $V^{\prime}\left(\operatorname{bod}_{\mathcal{Q}_{\varphi}^{\prime}}\right) \subseteq V\left(\operatorname{body}_{\mathcal{Q}_{\varphi}}\right)$. In particular, $\operatorname{Res}(1) \in V\left(\right.$ body $\left.y_{\mathcal{Q}_{\varphi}}\right)$. Then, valuations $V$ and $V^{\prime}$ agree on variables $x_{1}, \ldots, x_{m}, w_{1}, w_{0}$ because each atom in Fix is the only atom of $\mathcal{Q}_{\varphi}$ with its particular relation symbol. Similarly, the $\mathrm{YVal}_{i}$-atoms in $\mathcal{Q}_{\varphi}^{\prime}$ and $\mathcal{Q}_{\varphi}$ ensure that $V$ maps each pair $\left(y_{i}, \bar{y}_{i}\right)$ to $(0,1)$ or $(1,0)$. Let $\beta_{\mathbf{y}}$ be the truth assignment defined by $\beta_{\mathbf{y}}\left(y_{i}\right) \stackrel{\text { def }}{=} V\left(y_{i}\right)$, for every $i \in\{1, \ldots, n\}$. Since $\varphi \notin \Pi_{3}$-QBF, there is a truth assignment $\beta_{\mathbf{z}}$ such that $\left(\beta_{\mathbf{x}} \cup \beta_{\mathbf{y}} \cup \beta_{\mathbf{z}}\right) \not \forall \psi$. Let $V^{*}$ be the uniquely defined consistent 0-1-valued valuation induced by $\left(\beta_{\mathbf{x}} \cup \beta_{\mathbf{y}} \cup \beta_{\mathbf{z}}\right)$. Since $V^{*}$ is consistent, $V^{*}($ Circuit $) \subseteq V^{*}($ Gates $)$ and therefore $V^{*}\left(\right.$ body $\left._{\mathcal{Q}_{\varphi}}\right) \subseteq V\left(\right.$ body $\left._{\mathcal{Q}_{\varphi}}\right)$. Furthermore, since $\left(\beta_{\mathbf{x}} \cup \beta_{\mathbf{y}} \cup \beta_{\mathbf{z}}\right) \not \forall \psi$, we get $V^{*}\left(r_{k}\right)=0$ and therefore $\operatorname{Res}(1) \notin V^{*}\left(\operatorname{body}_{\mathcal{Q}_{\varphi}}\right)$ and, consequently, $V^{*}\left(\operatorname{bod}_{\mathcal{Q}_{\varphi}}\right) \subsetneq V\left(\operatorname{bod} y_{\mathcal{Q}_{\varphi}}\right)$, showing that $V$ is not minimal. 
(if) Let now $\varphi=\forall \mathbf{x} \exists \mathbf{y} \forall \mathbf{z} \psi(\mathbf{x}, \mathbf{y}, \mathbf{z})$ be a formula in $\Pi_{3}$-QBF and let $V^{\prime}$ be an arbitrary valuation for $\mathcal{Q}_{\varphi}^{\prime}$. We will show that there exists a minimal valuation $V$ for $\mathcal{Q}_{\varphi}$ such that $V^{\prime}\left(\operatorname{body}_{\mathcal{Q}_{\varphi}^{\prime}}\right) \subseteq V\left(\right.$ body $\left.\mathcal{Q}_{\varphi}\right)$, thus showing that parallel-correctness transfers from $\mathcal{Q}_{\varphi}$ to $\mathcal{Q}_{\varphi}^{\prime}$, again by Lemma 4.2 .

We assume in the following that all quantified variables appear (possibly negated) in $\psi$. Let $c_{0} \stackrel{\text { def }}{=} V^{\prime}\left(w_{0}\right)$ and $c_{1} \stackrel{\text { def }}{=} V^{\prime}\left(w_{1}\right)$. Since, neither $\mathcal{Q}_{\varphi}^{\prime}$ nor $\mathcal{Q}_{\varphi}$ uses any constant symbols, minimality of $V^{\prime}$ is not affected, if $V^{\prime}$ is composed with any bijection of the domain. The same holds for every valuation $V$ and the statement $V^{\prime}\left(\operatorname{body}_{\mathcal{Q}_{\varphi}^{\prime}}\right) \subseteq V\left(\right.$ body $\left._{\mathcal{Q}_{\varphi}}\right)$, as long as $V^{\prime}$ and $V$ are composed with the same bijection. Therefore, we can assume without loss of generality that $V^{\prime}\left(w_{0}\right)=0$ and $V^{\prime}\left(w_{1}\right) \in\{0,1\}$.

We distinguish three cases depending on whether $\operatorname{dom}\left(V^{\prime}\right) \subseteq\{0,1\}$ and $V^{\prime}\left(w_{1}\right)=1$.

Case $1\left(\operatorname{dom}\left(V^{\prime}\right) \subseteq\{0,1\}\right.$ and $\left.V^{\prime}\left(w_{1}\right)=1\right)$ : Let $\beta_{\mathbf{x}}$ be the partial truth assignment for the variables $x_{1}, \ldots, x_{m}$ in $\psi$ defined by $\beta_{\mathbf{x}}\left(x_{i}\right)=V^{\prime}\left(x_{i}\right)$, for every $i \in\{1, \ldots, m\}$. Since, $\varphi \in \Pi_{3}$-QBF, there exists a partial truth assignment $\beta_{\mathbf{y}}$ for the variables $y_{1}, \ldots, y_{n}$ in $\psi$ such that for each partial truth assignment $\beta_{\mathbf{z}}$ for the variables $z_{1}, \ldots, z_{p}$ we have $\left(\beta_{\mathbf{x}} \cup \beta_{\mathbf{y}} \cup \beta_{\mathbf{z}}\right) \models \psi$. For concreteness let $\beta_{\mathbf{z}}\left(z_{i}\right) \stackrel{\text { def }}{=} 0$, for $i \in\{1, \ldots, p\}$ and $\beta \stackrel{\text { def }}{=} \beta_{\mathbf{x}} \cup \beta_{\mathbf{y}} \cup \beta_{\mathbf{z}}$.

Let $V$ be the uniquely defined 0 -1-valued consistent valuation induced by $\beta$. Since $V$ is consistent it is also minimal by Claim C.8 and as $\beta \models \psi, V\left(r_{k}\right)=1$. Thanks to the latter, $V^{\prime}\left(\operatorname{body}_{\mathcal{Q}_{\varphi}^{\prime}}\right) \subseteq V\left(\right.$ body $\left.y_{\mathcal{Q}_{\varphi}}\right)$ follows easily and Case 1 is complete.

Case $2\left(\operatorname{dom}\left(V^{\prime}\right) \subseteq\{0,1\}\right.$ and $\left.V^{\prime}\left(w_{1}\right)=0\right)$ : Let $V$ be defined by

$$
V(u) \stackrel{\text { def }}{=} \begin{cases}V^{\prime}(u) & \text { if } u \in\left\{w_{0}, w_{1}, x_{1}, \ldots, x_{n}\right\} \\ 0 & \text { otherwise. }\end{cases}
$$

It is easy to see that $V^{\prime}\left(\operatorname{bod} y_{\mathcal{Q}_{\varphi}^{\prime}}\right) \subseteq V\left(\right.$ body $\left.y_{\mathcal{Q}_{\varphi}}\right)$. Furthermore, $V^{\prime}$ is minimal as every fact from $V\left(\right.$ body $\left.\mathcal{Q}_{\varphi}\right)$ either stems from an atom with (only) head variables or is in the unavoidable set $V$ (Gates).

Case 3 (For some $\mathbf{g}, \boldsymbol{V}^{\prime}\left(\boldsymbol{x}_{\boldsymbol{g}}\right) \notin\left\{\boldsymbol{c}_{\mathbf{0}}, \boldsymbol{c}_{\mathbf{1}}\right\}$ ): We recall that by our assumptions, $c_{0}=0=V^{\prime}\left(w_{0}\right)$ and $c_{1}=V^{\prime}\left(w_{1}\right) \in\{0,1\}$. The following argument works for both subcases, $c_{1}=1$ and $c_{1}=0$. We call a variable $x_{g}$ foul if $V^{\prime}\left(x_{g}\right) \notin\left\{c_{0}, c_{1}\right\}$. Likewise, we call a clause foul if it contains (positively or negatively) some foul variable. Let $G$ be the set of all indices $g$ for which $x_{g}$ is foul and $J$ be the set of all indices $j$ of foul clauses. Furthermore, let $10 a=V^{\prime}\left(x_{g}\right)$ for the minimal index $g \in G$.

We define valuation $V$ by

$$
V(u) \stackrel{\text { def }}{=} \begin{cases}V^{\prime}(u) & \text { if } u \in\left\{w_{0}, w_{1}, x_{1}, \ldots, x_{m}\right\}, \\ c_{1} & \text { if } u \in\left\{y_{1}, \ldots, y_{n}, z_{1}, \ldots, z_{p}\right\}, \\ c_{0} & \text { if } u \in\left\{\bar{y}_{1}, \ldots, \bar{y}_{n}, \bar{z}_{1}, \ldots, \bar{z}_{p}\right\}, \\ a & \text { if } x=\bar{x}_{g} \text { and } x_{g} \text { is foul, } \\ c_{0} & \text { if } x=\bar{x}_{g} \text { and } V^{\prime}\left(x_{g}\right)=c_{1}, \\ c_{1} & \text { if } x=\bar{x}_{g} \text { and } V^{\prime}\left(x_{g}\right)=c_{0} .\end{cases}
$$

For variables $s_{j}, V\left(s_{j}\right) \stackrel{\text { def }}{=} c_{1}$, if $C_{j}$ is foul or for all its literals $\ell$, it holds $V(\ell)=c_{1}$, otherwise $V\left(s_{j}\right) \stackrel{\text { def }}{=} c_{0}$. For variables $r_{j}, V\left(r_{j}\right) \stackrel{\text { def }}{=} c_{1}$, if $V\left(s_{i}\right)=c_{1}$, for some $i \leq j$ and $V\left(r_{j}\right) \stackrel{\text { def }}{=} c_{0}$, otherwise.

\footnotetext{
${ }^{10}$ In fact, any value not in $\left\{c_{0}, c_{1}\right\}$ would do.
} 
It is clear that $V^{\prime}\left(\operatorname{body}_{\mathcal{Q}_{\varphi}^{\prime}}\right) \subseteq V\left(\right.$ body $\left._{\mathcal{Q}_{\varphi}}\right)$ holds, but we can not expect that $V$ is minimal. There might be some And-facts in $V$ (Circuit) resulting from clauses that can be avoided by changing the valuation for some variables $z_{i}$. However, we can show in the following that every minimal valuation $V^{*}$ contained in $V$ fulfills $V^{\prime}\left(\operatorname{body}_{\mathcal{Q}_{\varphi}^{\prime}}\right) \subseteq V^{*}\left(\right.$ body $\left._{\mathcal{Q}_{\varphi}}\right)$ and thereby yields (C3).

To this end, let $V^{*}$ be a minimal valuation such that $V^{*} \leq_{\mathcal{Q}} V$. We show first that $V^{*}$ has to produce most facts from $V\left(\operatorname{body}_{\mathcal{Q}_{\varphi}}\right)$. This is immediate for all facts from $V\left(\left\{\mathrm{YVal}_{1}\left(y_{1}\right), \mathrm{YVal}_{1}\left(\bar{y}_{1}\right), \ldots, \mathrm{YVal}_{n}\left(y_{n}\right), \mathrm{YVal}_{n}\left(\bar{y}_{n}\right)\right\}\right), V($ Fix $)$, and $V($ Gates $)$.

Any facts of the form $V(\operatorname{Neg}(u, \bar{u}))$ that do not occur in $V($ Gates $)$ are of the form $\operatorname{Neg}\left(V^{\prime}\left(x_{g}\right), a\right)$, for some foul variable $x_{g}$. As $x_{g}$ occurs in the head, and there is at most one such fact per foul variable, these facts can not be avoided in $V^{*}\left(\operatorname{bod} y_{\mathcal{Q}_{\varphi}}\right)$. As all facts of the form $V^{*}(\operatorname{Neg}(u, \bar{u}))$ have to be in $V^{*}\left(b o d y_{\mathcal{Q}_{\varphi}}\right)$ and all variables $x_{i}, y_{i}$ occur in $h e a d_{\mathcal{Q}_{\varphi}}$, we can conclude that $V^{*}$ has to agree with $V$ for all variables of the form $x_{i}, \bar{x}_{i}, y_{i}, \bar{y}_{i}$ and on $w_{0}$ and $w_{1}$.

Therefore, it is clear for all facts from $V^{\prime}\left(b o d y_{\mathcal{Q}_{\varphi}^{\prime}}\right)$ except $\operatorname{Res}\left(c_{1}\right)$ that they are captured by $V^{*}\left(\right.$ body $\left.y_{\mathcal{Q}_{\varphi}}\right)$. It therefore only remains to show $\operatorname{Res}\left(c_{1}\right) \in V^{*}\left(\operatorname{body}_{\mathcal{Q}_{\mathscr{\ell}_{\mathrm{e}}}}\right)$.

Let $x_{g}$ be the foul variable that was used to define $a \stackrel{\text { def }}{=} V^{\prime}\left(x_{g}\right)$ and let $C_{j}$ be some clause in which it occurs. Thus, by definition of $V$ there is an And-fact in $V$ (Circuit) with value $a$ in one of its first three positions and with $c_{1}$ in its fourth position. Furthermore, all And-facts in $V$ (Circuit) with $a$-values have $c_{1}$ in their fourth position. Therefore, $V^{*}$ (Circuit) needs to contain at least one And-fact with $a$ in one of its first three positions and with $c_{1}$ in its fourth position. That is, $V^{*}\left(s_{i}\right)=c_{1}$, for at least one $i$. As $V^{*}($ Circuit $)$ can only contain Or-facts from $V($ Gates $)$, it follows that $V^{*}\left(r_{h}\right)=c_{1}$, for all $h \geq i$ and, in particular, for $h=k$. Therefore, $\operatorname{Res}\left(c_{1}\right) \in V^{*}\left(\operatorname{bod}_{\mathcal{Q}_{\varphi}}\right)$ and $V^{\prime}\left(\operatorname{body}_{\mathcal{Q}_{\varphi}^{\prime}}\right) \subseteq V^{*}\left(\operatorname{bod} y_{\mathcal{Q}_{\varphi}}\right)$.

\section{C.3 Proof of Lemma 4.10}

We define the following problem:

$\begin{array}{ll}\text { Problem: } & \text { StRonglyMinimal } \\ \text { Input: } & \text { CQ } \mathcal{Q} \\ \text { Question: } & \text { Is } \mathcal{Q} \text { strongly minimal? }\end{array}$

It remains to prove the following lemma:

Lemma C.9. Strongly Minimal is CoNP-hard.

Proof. The proof is by a reduction from the NP-complete 3-SAT problem to the complement of StronglyMinimal. To this end, let $\varphi$ be an input for 3-SAt. That is, a propositional formula in 3CNF with variables $x_{1}, \ldots, x_{m}$. Let $C_{1}, \ldots, C_{k}$ denote the (disjunctive) clauses in $\varphi$. We next construct the corresponding input instance $\mathcal{Q}_{\varphi}$ for StronglyMinimal.

The head of $\mathcal{Q}_{\varphi}$ is $H\left(w_{1}, w_{0}, x_{1}, \bar{x}_{1}, \ldots, x_{m}, \bar{x}_{m}\right)$. Intuitively, we represent Boolean values by pairs of variables: true is represented by $\left(w_{1}, w_{0}\right)$ and false is represented by $\left(w_{0}, w_{1}\right)$. Similarly, each literal of $\varphi$ is represented by a pair of variables. Formally, this representation is by a function rep, which maps literals from $\varphi$ to pairs of variables from $\mathcal{Q}$ via $\operatorname{rep}\left(x_{g}\right) \stackrel{\text { def }}{=}\left(x_{g}, \bar{x}_{g}\right)$ and $\operatorname{rep}\left(\neg x_{g}\right) \stackrel{\text { def }}{=}\left(\bar{x}_{g}, x_{g}\right)$, for each $g \in\{1, \ldots, m\}$. We extend rep to 3-clauses via $\operatorname{rep}\left(\left(\ell_{j, 1} \vee \ell_{j, 2} \vee \ell_{j, 3}\right)\right) \stackrel{\text { def }}{=}\left(y_{1}, y_{1}^{\prime}, y_{2}, y_{2}^{\prime}, y_{3}, y_{3}^{\prime}\right)$, where $\left(y_{i}, y_{i}^{\prime}\right)=\operatorname{rep}\left(\ell_{j, i}\right)$ for every $i \in\{1,2,3\}$. Besides the already mentioned head variables, $\mathcal{Q}_{\varphi}$ has only two non-head variables $r_{0}, r_{1}$.

Let $\mathbb{U}$ be the set of all 6 -tuples $\left(u_{1}, u_{1}^{\prime}, u_{2}, u_{2}^{\prime}, u_{3}, u_{3}^{\prime}\right)$, where $\left(u_{i}, u_{i}^{\prime}\right)$ equals either $\left(w_{1}, w_{0}\right)$ or $\left(w_{0}, w_{1}\right)$ for each $i \in\{1,2,3\}$, and let $\mathbb{U}^{+}=\mathbb{U} \backslash\left\{\left(w_{0}, w_{1}, w_{0}, w_{1}, w_{0}, w_{1}\right)\right\}$. Intuitively, $\mathbb{U}^{+}$represents all truth assignments that satisfy a three-way disjunction. 
We define body $\mathcal{Q}_{\varphi} \stackrel{\text { def }}{=}$ Values $\cup$ Cons $\cup \operatorname{Struct}(\varphi)$, where

- Values $\stackrel{\text { def }}{=}\left\{\operatorname{Val}\left(r_{0}, r_{1}\right), \operatorname{Val}\left(r_{1}, r_{0}\right)\right\}$ is a set of binary atoms restricting the possible mappings of variables $r_{0}, r_{1}$

- Cons $\stackrel{\text { def }}{=}\left\{\mathrm{C}_{j}\left(w_{1}, w_{0}, \mathbf{u}\right) \mid j \in\{1, \ldots, k\}, \mathbf{u} \in \mathbb{U}^{+}\right\}$is a set of 8-ary atoms encoding satisfying valuations of clauses; and,

- $\operatorname{Struct}(\varphi) \stackrel{\text { def }}{=}\left\{\mathrm{C}_{j}\left(r_{1}, r_{0}, \mathbf{y}_{j}\right) \mid j \in\{1, \ldots, k\}\right.$, where $\left.\mathbf{y}_{j} \stackrel{\text { def }}{=} \operatorname{rep}\left(C_{j}\right)\right\}$ is a set of 8-ary atoms representing the actual clauses of $\varphi$.

Correctness Obviously, $\mathcal{Q}_{\varphi}$ can be computed in polynomial time. It therefore only remains to show that $\mathcal{Q}_{\varphi} \notin$ StronglyMinimal if and only if $\varphi \in 3$-Sat.

(if) Let $\varphi \in 3$-SAt. We show that $\mathcal{Q}_{\varphi} \notin$ StronglyMinimal by constructing two valuations, that derive the same head fact, of which one requires strictly less facts than the other. By assumption, there is a truth assignment $\beta$ for variables $x_{1}, \ldots, x_{m}$ in $\varphi$ such that $\beta \models \varphi$.

Let $V^{\prime}$ and $V$ both be defined on the head variables as follows: $w_{0} \mapsto 0, w_{1} \mapsto 1$, and $x \mapsto \beta(x), \bar{x} \mapsto \overline{\beta(x)}$ for each $x \in\left\{x_{1}, \ldots, x_{m}\right\}$. The non-head variables are mapped for $V^{\prime}$ by $r_{0} \mapsto 0, r_{1} \mapsto 1$, and for $V$ by $r_{0} \mapsto 1, r_{1} \mapsto 0$.

Obviously, $V^{\prime}\left(\right.$ head $\left._{\mathcal{Q}_{\varphi}}\right)=V\left(\right.$ head $\left._{\mathcal{Q}_{\varphi}}\right)$. Since $\beta$ is a satisfying truth assignment for $\varphi$, it particularly satisfies every clause $C_{j}$ from $\varphi$. Therefore, for every $j \in\{1, \ldots, k\}$ there is some $\mathbf{u}_{j} \in \mathbb{U}^{+}$ such that $V^{\prime}\left(\mathrm{C}_{j}\left(r_{1}, r_{0}, \mathbf{y}_{j}\right)\right)=V^{\prime}\left(\mathrm{C}_{j}\left(w_{1}, w_{0}, \mathbf{u}_{j}\right)\right)$, which implies $V^{\prime}(\operatorname{Struct}(\varphi)) \subseteq V^{\prime}($ Cons $)$. Together with $V^{\prime}($ Values $)=V($ Values $)$ and $V^{\prime}($ Cons $)=V($ Cons $)$ this implies $V^{\prime}\left(\right.$ bod $\left._{\mathcal{Q}_{\varphi}}\right) \subseteq V\left(\right.$ body $\left.\mathcal{Q}_{\varphi}\right)$. Because $V\left(r_{1}, r_{0}\right)=(0,1) \neq(1,0)=V\left(w_{1}, w_{0}\right)$, it holds $V(\operatorname{Struct}(\varphi)) \nsubseteq V V($ Cons $)$, which further implies $V^{\prime}\left(\right.$ body $\left._{\mathcal{Q}_{\varphi}}\right) \subsetneq V\left(\right.$ body $\left._{\mathcal{Q}_{\varphi}}\right)$. Thus, $V^{\prime}$ contradicts minimality of $V$ and therefore we have $\mathcal{Q}_{\varphi} \notin$ STRONGLYMinimaL.

(only-if) Now, let $\varphi$ be unsatisfiable. We show that $\mathcal{Q}_{\varphi} \in$ StronglyMinimal. Actually, we prove that there are even no two different valuations $V$ and $V^{\prime}$ for $\mathcal{Q}_{\varphi}$ for which $V^{\prime} \leq_{\mathcal{Q}_{\varphi}} V$.

Towards a contradiction, assume that $V$ and $V^{\prime}$ are two different valuations for $\mathcal{Q}_{\varphi}$ with $V^{\prime}\left(\right.$ head $\left.\mathcal{Q}_{\varphi}\right)=$ $V\left(\right.$ head $\left._{\mathcal{Q}_{\varphi}}\right)$ and $V^{\prime}\left(\operatorname{body}_{\mathcal{Q}}\right) \subseteq V\left(\operatorname{body}_{\mathcal{Q}}\right)$. By design of the set Values $=\left\{\operatorname{Val}\left(r_{0}, r_{1}\right), \operatorname{Val}\left(r_{1}, r_{0}\right)\right\}$, one of the following holds: (1) $V^{\prime}\left(r_{0}\right)=V\left(r_{0}\right)$ and $V^{\prime}\left(r_{1}\right)=V\left(r_{1}\right)$; or $(2) V^{\prime}\left(r_{0}\right)=V\left(r_{1}\right)$ and $V^{\prime}\left(r_{1}\right)=V\left(r_{0}\right)$. From our assumption $V^{\prime} \neq V$ and since $r_{0}, r_{1}$ are the only non-head-variables, we can conclude that (2) must hold and that in addition $V\left(r_{0}\right) \neq V\left(r_{1}\right)$.

To obtain a contradiction, we next show that there is a $C_{j}$-atom $A \in \operatorname{Struct}(\varphi)$ for which $V^{\prime}(A) \notin$ $V\left(\right.$ body $\left.\mathcal{Q}_{\varphi}\right)$ implying that $V^{\prime}\left(\operatorname{bod} y_{\mathcal{Q}_{\varphi}}\right) \nsubseteq V\left(\operatorname{bod} y_{\mathcal{Q}_{\varphi}}\right)$. In fact, it already suffices to show that $V^{\prime}(A) \notin$ $V($ Cons $)$, since $A$ cannot be mapped to any fact in $V(\operatorname{Struct}(\varphi))$ because of the different mapping of variables $r_{0}, r_{1}$.

Let $c_{0} \stackrel{\text { def }}{=} V\left(w_{0}\right)$ and $c_{1} \stackrel{\text { def }}{=} V\left(w_{1}\right)$. We distinguish two cases.

Case $1\left(V\left(r_{1}, r_{0}\right)=\left(c_{1}, c_{0}\right)\right)$ : Let $A \stackrel{\text { def }}{=} \mathrm{C}_{j}\left(r_{1}, r_{0}, \mathbf{y}_{j}\right)$ be an arbitrary $\mathrm{C}_{j}$-atom. Then $V^{\prime}\left(\mathrm{C}_{j}\left(r_{1}, r_{0}, \mathbf{y}_{j}\right)\right) \notin$ $V($ Cons $)$ because $V^{\prime}\left(r_{1}, r_{0}\right) \neq V\left(w_{1}, w_{0}\right)$.

Case $2\left(V\left(r_{1}, r_{0}\right)=\left(c_{0}, c_{1}\right)\right)$ :

We can assume that $V^{\prime}\left(x_{g}, \bar{x}_{g}\right) \in\left\{\left(c_{0}, c_{1}\right),\left(c_{1}, c_{0}\right)\right\}$ for every $g \in\{1, \ldots, m\}$. Otherwise, existence of $A$ follows immediately, as $V$ (Cons) only uses values $c_{0}, c_{1}$. 
Therefore, valuation $V^{\prime}$ induces a truth assignment $\beta$ by $x \mapsto 0$, if $V(x)=c_{0}$, and $x \mapsto 1$, if $V(x)=c_{1}$, for every $x \in\left\{x_{1}, \ldots, x_{m}\right\}$. Since $\varphi$ is not satisfiable, there is some $j$ such that $\beta \not C_{j}$. Thus, we have $\beta\left(\ell_{j, 1}, \ell_{j, 2}, \ell_{j, 3}\right)=(0,0,0)$ and $V^{\prime}\left(\mathbf{y}_{j}\right)=\left(c_{0}, c_{1}, c_{0}, c_{1}, c_{0}, c_{1}\right) \notin \mathbb{U}^{+}$.

Therefore, valuation $V^{\prime}$ cannot map atom $A=\mathrm{C}_{j}\left(r_{0}, r_{1}, \mathbf{y}_{j}\right)$ from $\operatorname{Struct}(\varphi)$ to any fact in $V($ Cons $)$.

\section{Proofs for Section 5: Families of Distribution Policies}

\section{D.1 Proof of Proposition 5.4}

We provide two reductions from an NP-complete problem to the problem of deciding condition (C3) on arbitrary queries $\mathcal{Q}^{\prime}$ and $\mathcal{Q}$. In the first reduction, given in Proposition D.1, $\mathcal{Q}$ is acyclic while $\mathcal{Q}^{\prime}$ is acyclic in the second reduction, given in Proposition D.2. Clearly, Proposition [5.4 follows from Proposition D.2 and Proposition D.1. That the proposition holds even for full $\mathcal{Q}$ follows from the fact that the head of $\mathcal{Q}$ is irrelevant for the truth of condition (C3). That is, e.g., in the proof of Proposition D.2 the head of $\mathcal{Q}$ could be chosen full.

For a CQ $\mathcal{Q}$, we denote by $\mathcal{H}_{\mathcal{Q}}$ the hypergraph in which every node corresponds to a variable in $\mathcal{Q}$, and there is a hyperedge between a set of nodes in $\mathcal{H}_{\mathcal{Q}}$ if the corresponding variables of $\mathcal{Q}$ occur together in some atom in the body of $\mathcal{Q}$. For the definition of acyclicity, we use the well-known GYO reduction [2]. In particular, a query $\mathcal{Q}$ is acyclic if repeatedly removing nodes in $\mathcal{H}_{\mathcal{Q}}$ that are in only one hyperedge and all the hyperedges that are contained in another hyperedge, results in the empty hyperedge.

For both cases, NP-hardness relies on a reduction from the NP-complete graph 3-colorability problem, which asks for a given undirected graph $G=(V, E)$, whether there exists a mapping $h$ that assigns to every node in $G$ a color from the set $C=\{r, g, b\}$, such that a different color is assigned to every pair of adjacent nodes. Hereafter we will refer to such a mapping $h$ for $G$ as a 3-color assignment or mapping for $G$.

For ease of exposition, we assume $V \subseteq \operatorname{var}, C \subseteq \operatorname{var}$ (where $V$ and $C$ are disjoint sets). Further, by $E_{C}$ we denote the set of all pairs $(c, d) \in C^{2}$ where $c \neq d$. Intuitively, these pairs correspond to possible valid colorings of edges.

Proposition D.1. For Boolean CQs $\mathcal{Q}$ and $\mathcal{Q}^{\prime}$, where $\mathcal{Q}$ is acyclic, it is NP-hard to decide whether condition (C3) holds.

Proof. As mentioned above, we reduce from the NP-complete graph 3-colorability problem. To this end, let $G=(V, E)$ be an arbitrary input graph.

Based on $G$, we construct a query $\mathcal{Q}^{\prime}$ and $\mathcal{Q}$ as follows 11

$$
\begin{aligned}
Q^{\prime} & :() \leftarrow \bigwedge_{(x, y) \in E} E(x, y), \bigwedge_{(c, d) \in E_{C}} E(c, d), \operatorname{Fix}(r, g, b) . \\
Q:() & \leftarrow \bigwedge_{(c, d) \in E_{C}} E(c, d), \operatorname{Fix}(r, g, b) .
\end{aligned}
$$

Here, both $\mathcal{Q}$ and $\mathcal{Q}^{\prime}$ are defined over the schema $\{E$, Fix $\}$ where $E$ is binary and Fix is ternary. Notice in particular that the second part of the body of $\mathcal{Q}^{\prime}$ corresponds exactly to body $y_{\mathcal{Q}}$. Furthermore, notice that $\mathcal{Q}^{\prime}$ and $\mathcal{Q}$ can be computed in time polynomial in the size of $G$.

\footnotetext{
${ }^{11}$ The construction is inspired by the construction given in 8 to show NP-completes for the folding problem.
} 
Claim. There is a 3-color assignment for $G$ if and only if there is a simplification $\theta$ for $\mathcal{Q}^{\prime}$ and a substitution $\rho$ for $\mathcal{Q}$ such that $\operatorname{body}_{\theta\left(\mathcal{Q}^{\prime}\right)} \subseteq \operatorname{bod} y_{\rho(\mathcal{Q})}$.

(if) Suppose there is a simplification $\theta$ for $\mathcal{Q}^{\prime}$ and a substitution $\rho$ for $\mathcal{Q}$, such that $\operatorname{body}_{\theta\left(\mathcal{Q}^{\prime}\right)} \subseteq$ body $y_{(\mathcal{Q})}$. By definition of simplification, body ${ }_{\theta\left(\mathcal{Q}^{\prime}\right)} \subseteq$ bod $_{\mathcal{Q}^{\prime}}$. In particular, $\theta(\operatorname{Fix}(r, g, b)) \subseteq b o d y_{\mathcal{Q}^{\prime}}$, so it must be that $\theta(\operatorname{Fix}(r, g, b))=\operatorname{Fix}(r, g, b)$. So, $\operatorname{Fix}(r, g, b)$ must be in $\operatorname{bod} y_{\rho(\mathcal{Q})}$, implying that $\operatorname{bod}_{\rho(\mathcal{Q})}=$ body $y_{\mathcal{Q}}$ as $\mathcal{Q}$ contains only colors. This means that $\theta$ maps every node in $V$ onto a color in $C$. Specifically, by construction of $\mathcal{Q}^{\prime}$ and $\mathcal{Q}$, every two adjacent nodes in $G$ are mapped by $\theta$ onto distinct colors in $C$. Now, let $h$ be the mapping defined as $h(x)=\theta(x)$ for $x \in V$. Then, by the above, $h$ is a 3 -color assignment for $G$.

(only-if) Suppose there is a 3-color assignment $h$ for $G$. Let $\theta$ be the mapping defined as $\theta(x)=h(x)$ for $x \in V$ and $h(c)=c$ for $c \in C$. As $h$ is a 3-color assignment, body $y_{\theta\left(\mathcal{Q}^{\prime}\right)} \subseteq \operatorname{bod}_{\mathcal{Q}}$. As there are no head variables, it readily follows that $\theta$ is a simplification. Taking $\rho$ as the identity for $C$, it follows that $\operatorname{bod}_{\theta\left(\mathcal{Q}^{\prime}\right)} \subseteq \operatorname{body}_{\mathcal{Q}}=\operatorname{bod} y_{\rho(\mathcal{Q})}$.

Acyclicity of $\mathcal{Q}$. By construction of $\mathcal{Q}$, vars $\left(\right.$ body $\left.y_{\mathcal{Q}}\right) \subseteq C$. So, every hyperedge in $\mathcal{H}_{\mathcal{Q}}$ is contained in the hyperedge that represents atom $\operatorname{Fix}(r, g, b)$, implying that all the other hyperedges can be removed immediately. Hereafter, only one hyperedge remains (i.e, the hyperedge that represents $\operatorname{Fix}(r, g, b)$ ), implying that all the remaining nodes can be removed, which results in the empty hyperedge. Hence, $\mathcal{Q}$ is acyclic.

Proposition D.2. For Boolean CQs $\mathcal{Q}$ and $\mathcal{Q}^{\prime}$, where $\mathcal{Q}^{\prime}$ is acyclic, it is NP-hard to decide whether condition (C3) holds.

Proof. We give a reduction from the NP-complete graph 3-colorability problem. Let $G=(V, E)$ be an undirected graph, where $m=|E|$. For ease of presentation, we assume that every edge is represented by only one tuple in $E$, i.e., that $(x, y) \in E$ implies $(y, x) \notin E 12$.

Further, we consider a labeling function $\ell$ that assigns to every edge in $G$ a unique label in var $\backslash(V \cup C)$. Let $Z=i m g(\ell)=\left\{z_{1}, \ldots, z_{m}\right\}$. Let $W=\left\{w_{z, i} \mid z \in Z, i \in\{1, \ldots, 10 m\}\right.$. We assume $W$ is disjoint from $V$, $C$, and $Z$. In the construction we represent edges of $G$ as atoms over the ternary relation $E$, where the first variable denotes its associated label, the second and third variable denote its end nodes.

Based on $G$, we construct queries $\mathcal{Q}^{\prime}$ and $\mathcal{Q}$ as follows:

$$
\begin{aligned}
& \mathcal{Q}^{\prime}:() \leftarrow \bigwedge_{(c, d) \in E_{C}, z \in Z} E(z, c, d), \bigwedge_{i \in\{1, \ldots, m-1\}} \operatorname{Fix}\left(z_{i}, z_{i+1}, r, g, b\right) . \\
& \mathcal{Q}:() \leftarrow \bigwedge_{(x, y) \in E} E(\ell(x, y), x, y), \bigwedge_{z \in Z, i \in\{1,3,5,7,9\}} E\left(z, w_{z, i}, w_{z, i+1}\right), \bigwedge_{i \in\{1, \ldots, m-1\}} \operatorname{Fix}\left(z_{i}, z_{i+1}, r, g, b\right) .
\end{aligned}
$$

Notice that $\mathcal{Q}^{\prime}$ represents for every edge in $G$ all the potentially valid color-assignments within the range $C$, implying $6 E$-atoms for each edge in $G$, whereas $\mathcal{Q}$ has for every edge in $G$, one $E$-atom that represents the edge and 5 additional $E$-atoms, containing unique variables on the end-node positions. Hereafter, we will refer to the latter as free $E$-atoms.

Claim. There is a simplification $\theta$ for $\mathcal{Q}^{\prime}$ and a substitution $\rho$ for $\mathcal{Q}$ such that body $y_{\theta\left(\mathcal{Q}^{\prime}\right)} \subseteq$ body $y_{\rho(\mathcal{Q})}$ if and only if there is a 3-color assignment for $G$.

\footnotetext{
${ }^{12}$ Notice that this requires to choose a direction for every edge, but that no information gets lost. The choice itself is of no further concern and can be implemented in an arbitrary fashion.
} 
(if) Let $h$ be a valid 3-color assignment for $G$. We show that there is a simplification $\theta$ for $\mathcal{Q}^{\prime}$ and substitution $\rho$ for $\mathcal{Q}$ such that $\operatorname{body}_{\theta\left(\mathcal{Q}^{\prime}\right)} \subseteq \operatorname{bod} y_{\rho(\mathcal{Q})}$. Let $\theta$ be the identity simplification for $\mathcal{Q}^{\prime}$. Let $\rho^{\prime}$ be the partial substitution for $\mathcal{Q}$ that is the identity on edge labels and colors, and maps the variables in $V$ onto variables in $C$ consistent with $h$. That is, $\rho^{\prime}(x)=x$ for $x \in C \cup Z$ and $\rho^{\prime}(x)=h(x)$ for $x \in V$. Now, $\rho^{\prime}$ already guarantees that every extension of $\rho^{\prime}$ satisfies the containment property body $y_{\mathcal{Q}^{\prime}} \subseteq \rho^{\prime}\left(\right.$ body $\left.y_{\mathcal{Q}}\right)$ for all the Fix-atoms in $\mathcal{Q}^{\prime}$ and for exactly $m E$-atoms in $\mathcal{Q}^{\prime}$ (all having a distinct edge label). Next, we extend $\rho^{\prime}$ to a complete substitution $\rho$ by mapping the variables in $W$ in such a way that the $5 m$ free $E$-atoms are mapped on exactly the $5 m$ remaining $E$-atoms in $\mathcal{Q}^{\prime}$. Notice that such an extension exists because the variables in $W$ are all unique for $\mathcal{Q}$.

(only-if) Let $\theta$ be a simplification for $\mathcal{Q}^{\prime}$ and $\rho$ a substitution for $\mathcal{Q}$, where $\operatorname{body} y_{\theta\left(\mathcal{Q}^{\prime}\right)} \subseteq \operatorname{body}{ }_{\rho(\mathcal{Q})}$. We show that there is a valid 3-color assignment for $G$. The proof proceeds under the assumption that

$$
\operatorname{bod}_{\theta\left(\mathcal{Q}^{\prime}\right)}=\operatorname{body}_{\mathcal{Q}^{\prime}}
$$

We prove below that $(\dagger)$ holds. Assuming $(\dagger)$, it follows that body $y_{\mathcal{Q}^{\prime}} \subseteq$ body $y_{\rho(\mathcal{Q})}$. Let $h$ be the mapping defined by $h(x)=\rho(x)$ for every $x \in V$. To show that $h$ is a valid 3-color mapping for $G$, we argue that $i m g(h) \subseteq C$ and $h(x) \neq h(y)$ for every two adjacent nodes $x, y$ in $G$. We observe that for $\rho$ to satisfy $\operatorname{body}_{\mathcal{Q}^{\prime}} \subseteq \operatorname{body}_{\rho(\mathcal{Q})}, \rho$ must be such that every $E$-atom in the body of $\mathcal{Q}^{\prime}$ is mapped onto an $E$-atom in the body of $\mathcal{Q}$. Then, by construction of $\mathcal{Q}^{\prime}$ and $\mathcal{Q}$ (both contain precisely six $E$-atoms per edge label $z$ ) it follows that every variable in $V$ is mapped by $\rho$ onto a variable in $C$. In particular, for every edge-representing atom in $\mathcal{Q}, \rho(E(z, x, y))=E\left(z^{\prime}, c, d\right)$, where $c \neq d$. So, $\operatorname{img}(h) \subseteq C$, and $h(x) \neq h(y)$ for every two adjacent nodes in $V$, implying $h$ to be a valid 3-color mapping for $G$.

It remains to show $(\dagger)$. We actually prove a stronger result, namely that $\theta$ must be the identity simplification. By definition of simplification, $\operatorname{bod} y_{\theta\left(\mathcal{Q}^{\prime}\right)} \subseteq \operatorname{bod} y_{\mathcal{Q}^{\prime}}$. In particular, $\theta\left(\operatorname{Fix}\left(z_{i}, z_{i+1}, r, g, b\right)\right) \subseteq$ body $y_{\mathcal{Q}^{\prime}}$ for every $i \in\{1, \ldots, m-1\}$. As the Fix-atoms in $\mathcal{Q}^{\prime}$ all agree on the last three variables, it follows that $\theta$ is the identity on the variables $r, g, b$. It remains to show that $\theta$ is also the identity on the edge labels. Towards a contradiction suppose that one of the edge labels in $\mathcal{Q}^{\prime}$ is mapped onto another variable by $\theta$. Then, by construction of $\mathcal{Q}^{\prime}$, it must be that this other variable is also an edge-label. Therefore, let $z_{i}$ be such a variable, where $\theta\left(z_{i}\right)=z_{j}$, for $i, j \in\{1, \ldots, m\}$ and $i \neq j$. Hereafter, by Fix ${ }_{k}$ we denote the atom $\operatorname{Fix}\left(z_{k}, z_{k+1}, r, g, b\right)$, where $1 \leq k \leq m-1$.

We distinguish two cases:

1. Case $(i<j)$ : By the assumption $\theta\left(z_{i}\right)=z_{j}$ and the definition of simplification (i.e., $\left.\theta\left(\operatorname{Fix}_{i}\right) \in b d_{\mathcal{Q}^{\prime}}\right)$, it must be that $\theta\left(\operatorname{Fix}_{i}\right)=\operatorname{Fix}_{j}$ (because $\operatorname{Fix}_{j}$ is the only atom in $\mathcal{Q}^{\prime}$ where variable $x_{j}$ occurs on the first position). So, $\theta\left(x_{i+1}\right)=\theta\left(x_{j+1}\right)$. We can repeat the above argument to show that $\theta\left(\operatorname{Fix}_{i+1}\right)=\operatorname{Fix}_{j+1}$, and in general, $\theta\left(\operatorname{Fix}_{i+k}\right)=\operatorname{Fix}_{j+k}$ for every positive integer $k \leq m-j-1$. Notice that $\theta\left(\operatorname{Fix}_{m-j-1}\right)=$ Fix $_{m-1}$, i.e., it is the last atom of the chain. Further, $\theta\left(z_{m-j}\right)=z_{m}$. Now, because $\theta\left(\right.$ bod $\left._{\mathcal{Q}^{\prime}}\right) \subseteq$ body ${ }_{\mathcal{Q}^{\prime}}$, it must be that $\theta\left(\operatorname{Fix}_{m-j-1}\right) \subseteq \operatorname{bod} y_{\mathcal{Q}^{\prime}}$, requiring that there is an atom in body $y_{\mathcal{Q}^{\prime}}$ with $z_{m}$ on the first position. By construction of $\mathcal{Q}^{\prime}$ there is no such atom.

2. Case $(i>j)$ : The proof is analogous to the proof for case (1), except that we show an argument in the reverse direction, towards an atom where $z_{1}$ is on the second position.

Acyclicity of $\mathcal{Q}$. Again, acyclicity can be verified by a straightforward GYO reduction on the hypergraph $\mathcal{H}_{\mathcal{Q}^{\prime}}$. Notice that all the hyperedges that represent an $E$-atom for $\mathcal{Q}^{\prime}$ are contained in the hyperedges that 
represent the Fix-atoms for a matching edge label. So, all the hyperedges representing an $E$-atom can be removed immediately. Now, the proof proceeds by induction on the number of Fix-atoms. For the base case, assume there is only one Fix-atom. Then all variables occur in only one hyperedge and thus can be removed which results in the empty hyperedge. Next, assume that $\mathcal{Q}^{\prime}$ is acyclic when it has a chain of at most $i$ Fix-atoms. For the induction step we assume that there are $i+1$ Fix-atoms. W.l.o.g., we assume that the first variable in the chain is $z_{1}$, and the last is $z_{i+2}$. Notice that $z_{1}$ is in only one hyperedge, namely the hyperedge that represents Fix $\left(z_{1}, z_{2}, r, g, b\right)$. After removing $z_{1}$, this hyperedge is entirely contained by the hyperedge representing the next Fix-atom, i.e., $\operatorname{Fix}\left(z_{2}, z_{2}, r, g, b\right)$. Consequently, the hyperedge that represents $\operatorname{Fix}\left(z_{1}, z_{2}, r, g, b\right)$ is eliminated. The resulting hypergraph now represents a chain of $i$ Fix-atoms. Hence, the result follows from the induction hypothesis.

Remark D.3. As Proposition D.1 shows NP-completeness when $\mathcal{Q}$ is acyclic and Proposition D.2 shows NP-completeness when $\mathcal{Q}^{\prime}$ is acyclic, it is an interesting question whether it is still NP-complete to decide condition (C3) if both $\mathcal{Q}$ and $\mathcal{Q}^{\prime}$ are acyclic. When allowing arbitrary arity relations, acyclicity is easily achieved by the use of one atom that contains all variables of the query. Particularly, one can add to $\mathcal{Q}$ in the reduction for Proposition D.2 an atom $A$ that contains every variable of $\mathcal{Q}$. Then, the proof remains valid as-is, but both $\mathcal{Q}^{\prime}$ and $\mathcal{Q}$ are acyclic. Nevertheless, under bounded-arity database schemas, the problem remains open. 